\title{
Ionization rate coefficients for the elements hydrogen through zinc ${ }^{\star}$
}

\begin{abstract}
K. P. Dere
George Mason University, 4400 University Dr., Fairfax VA, 22030, USA

e-mail: kdere@gmu.edu

Received 9 November 2006 / Accepted 7 February 2007

\section{ABSTRACT}

Aims. The interpretation of astrophysical spectra depends directly on a knowledge of the ionization state of the emitting plasma. This is determined, in part, from collisional ionization rate coefficients. The most recent assessments of these were performed by Arnaud \& Rothenflug (1985, A\&AS, 60, 425) and Arnaud \& Raymond (1992, ApJ, 398, 394). Since their work, new laboratory measurements of ionization cross sections have become available as well as the Flexible Atomic Code (FAC) which enables theoretical calculations of these rates. Our goal is to provide a complete set of ionization rate coefficients for the elements hydrogen through zinc.

Methods. A scaling law, which assists the analysis of ionization cross sections and rate coefficients, has been developed following the approach of Burgess \& Tully (1992, A\&A, 254, 436). Essentially all available measured cross sections along each isoelectronic sequence have been examined and compared to cross sections calculated with the Flexible Atomic Code (FAC) and with other calculations. Two approaches has been taken to provide a complete set of ionization cross sections. In the first, fits to scaled measured ionization cross sections, particularly for neutral and singly ionized species, are performed. In the second, fits to scaled calculated direct ionization and excitation-autoionization cross sections are performed to provide the remainder of the set. The fits to the cross sections are then integrated over a Maxwellian velocity distribution to derive ionization rate coefficients.

Results. A complete set of ground level ionization cross sections and rate coefficients has been developed through the combination of these two approaches. A tabulation of parameter fits to ground level ionization rate coefficients for all atoms and ions of the elements of $\mathrm{H}$ through $\mathrm{Zn}$ is provided.
\end{abstract}

Key words. atomic data - atomic processes - plasmas

\section{Introduction}

The interpretation of astrophysical spectra depends directly on a knowledge of the ionization state of the observed plasma. In a collisionally dominated plasma, such as the solar corona, the ionization state is determined by a balance of the electron collisional ionization rates and the radiative and dielectronic recombination rates. Whether the plasma is considered to be in a steady or transient state, it is necessary to have an accurate knowledge of these rates. This paper describes a new complete set of ionization rate coefficients for all ions of all elements from hydrogen through zinc based on the most recently available laboratory measurements and theoretical calculations.

Prior to this work, the most recent analysis of ionization rates was performed by Arnaud \& Rothenflug (1985) and by Arnaud \& Raymond (1992) for iron. Since that time, a number of new laboratory measurements have been performed and reported and the Flexible Atomic Code (FAC) has been developed by $\mathrm{Gu}$ (2002) to enable calculations of ionization cross sections. In addition, the use of scaling laws, such as those developed by Burgess \& Tully (1992) for collisional excitation, have proven useful. In many important respects, this analysis follows the earlier analyses of Arnaud \& Rothenflug (1985) and Arnaud \& Raymond (1992) with a strong reliance on both observational and theoretical results.

* Full Table 29 is only available in electronic form at the CDS via anonymous ftp to cdsarc.u-strasbg.fr (130.79.128.5) or via http://cdsweb.u-strasbg.fr/cgi-bin/qcat?J/A+A/466/771
The result of this analysis is a set of spline fits to scaled temperatures and scaled ionization rate coefficients that are available electronically. Further, the cross sections and rate coefficients will be available through the CHIANTI atomic database for astrophysical spectroscopy Dere et al. (1997), Landi et al. (2006).

\section{Approach}

Ionization caused by the collision of electrons with neutral and ionized species occurs primarily through two mechanisms. The first is direct ionization (DI) where a bound electron is converted to a free electron in the collision. The second process is usually referred to as "excitation-autoionization" (EA). Here, a bound electron is excited into an autoionizing state. At this point, there is a competition between a stabilizing radiative transition and an autoionizing transition. The relative contributions of the two processes can vary considerably with isoelectronic sequence. For the hydrogen through neon isoelectronic sequences, direct ionization dominates. For more complex ions, EA is often the dominant ionization process.

\subsection{Empirical scaling laws for ionization}

Lotz (1967a) developed the "classical" ionization scaling, where the scaled cross section is given by cross section multiplied by the square of the ionization potential and the scaled energy is given by incident electron energy divided by the ionization potential. The importance of this scaling is that classically 
scaled ionization cross section plotted versus scaled energy varies slowly along an isoelectronic sequence, particularly at high values of the nuclear charge $\mathrm{Z}$.

Bethe (1930) showed that at high energy, the ionization cross section scales as $\sigma \sim C \log u / u$ where $\sigma$ is the direct ionization cross section, $\mathrm{u}$ is the scaled energy $E / I, E$ is the energy of the incident electron and $I$ is the ionization potential. The constant $C$ is the Bethe coefficient and is give by Younger (1981c)

$C=\frac{I}{\pi \alpha} \int \frac{\sigma_{\mathrm{PI}}}{\epsilon} \mathrm{d} \epsilon$

where $\alpha$ is the fine structure constant, $\sigma_{\mathrm{PI}}$ is the photoionization cross section and $\epsilon$ is the photon energy. The photoionization cross sections of Verner \& Yakovlev (1995) have been used to calculation the Bethe coefficient for all of the ionization cross sections reported here. Further, the collision strength, a component of the EA rate, also approaches a high energy limit $\Omega \sim(4 g f / \Delta E) \log u$ where $g f$ is the weighted oscillator strength and $\Delta E$ the excitation energy Burgess \& Tully (1992).

The CHIANTI database has made extensive use of the scaling laws that Burgess \& Tully (1992) developed for collisional excitation. A similar scaling law for ionization cross sections has been developed here. It is based on a combination of the classical cross section scaling of Lotz (1967a), the Bethe (1930) limit for direct ionization and the Burgess \& Tully (1992) scaling for allowed transitions. The scaling involves a scaled energy $U$,

$U=1-\frac{\log f}{\log (u-1+f)}$

and a scaled cross section $\Sigma$ in a manner similar to Burgess \& Tully (1992),

$\Sigma=\frac{u \sigma I^{2}}{\log (u)+1}$.

Here $f$ is an adjustable parameter. This type of scaling will be referred to as BT scaling and has several advantages. The energy range from threshold to infinity is mapped onto a finite scale from zero to unity. The extended high energy range is highly compressed while the energy range just above threshold is expanded. The scaled cross section $\Sigma$ approaches a known constant at high energy and for fitting cross sections, the threshold value for $\Sigma$ for $U=0(u=1)$ can be set to zero. As with classically scaled cross sections, the BT cross section scaling approaches a fixed function as one proceeds along an isoelectronic sequence, at least in the case of direct ionization.

Figure 1 shows an example of this approach as applied to measurements of $\mathrm{Li}$ II. This ion contains only 2 electrons in the 1s shell so that the direct cross section should not show any structure resulting from ionization from different shells. The measurements extend to nearly 400 times the threshold value. The cross sections at the highest energies are from the measurements of Peart \& Dolder (1968) who sought to verify the high energy scaling. The other measurements are from Lineberger et al. (1966), Peart et al. (1969b), and Wareing (1967). The fit to the experimental data is shown for classical scaling (top) and for BT scaling (bottom). It can be seen that the ionization cross section does approach a constant value at BT scaled energies of about 0.8 but this that value is about $40 \%$ higher than the high-energy Bethe limit. In fact, the measured cross sections have often been found to approach a high-energy limit that is a factor of 1.4 to 2.0 higher than the Bethe limit. Nevertheless, the Bethe limit has been used for all of the ionization cross section fits.
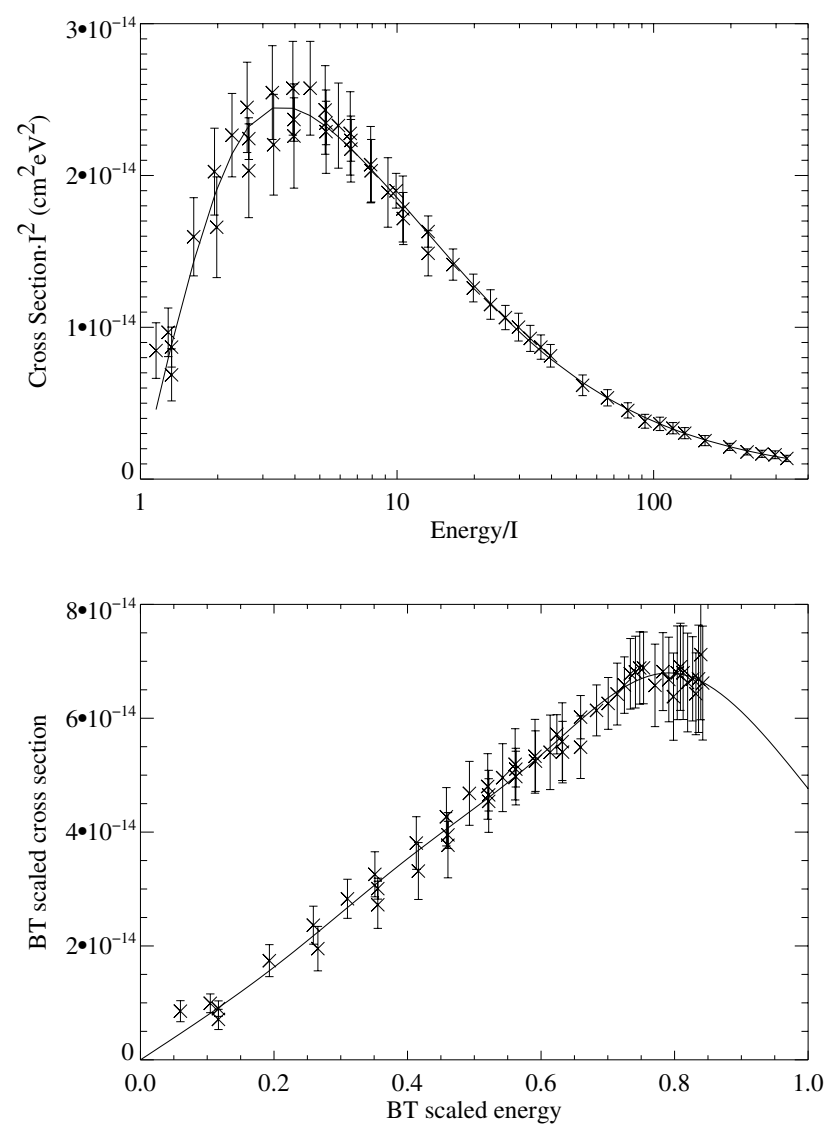

Fig. 1. Top: classically scaled ionization cross section for Li II. Bottom: BT scaled cross section versus scaled energy and the fit to the data.

One of the techniques used here to obtain ionization cross sections over the entire range of energies is to derive a spine fit to the BT scaled measurements. As this procedure results in a smoothly varying curve, it is only used for cases where there is little apparent structure in the measured cross sections. Typically this is the case for neutrals and singly ionized species.

\subsection{Calculation of ionization cross sections}

FAC has been used to calculate the DI cross sections from inner and outer shells of the various ions as explained later in the text. The ionization potentials are taken from the CHIANTI database, which in turn, are largely based on values from the NIST database Ralchenko et al. (2006). Cross sections for excitation autoionization have also been calculated with FAC. This involved solving the atomic structure, calculating collision strengths into auto-ionizing levels and determining the branching ratio for ionization. All of the rates have been summed into a reduced set and fit with a Burgess \& Tully (1992) scaling, including a value for the high energy limit. The FAC distorted wave calculations tend to be most accurate for doubly ionized species and higher. The use of scaling law fits to the cross sections of neutrals and singly-ionized ions is often the most accurate approach.

\subsection{Error estimates}

Estimates of the errors in the cross sections are provided, as in Table 1. The column labeled Experimental error lists the total error in the individual experimental measurements. The column 
Table 1. Ionization cross section measurements for the hydrogen isoelectronic sequence.

\begin{tabular}{llll}
\hline \hline Ion & Data source & \multicolumn{2}{l}{ Experimental error Final error } \\
\hline H I & Shah et al. (1987) & $7 \%$ & $7 \%$ \\
He II & Achenbach et al. (1984) & $10 \%$ & $15 \%$ \\
& Defrance et al. (1981a) & - & \\
& Dolder et al. (1961) & $25 \%$ & \\
& Peart et al. (1969b) & $12 \%$ & \\
Li III & Tinschert et al. (1989a) & $10 \%$ & $10 \%$ \\
B v & Aichele et al. (1998) & $10 \%$ & $10 \%$ \\
C VI & Aichele et al. (1998) & $10 \%$ & $10 \%$ \\
N VII & Aichele et al. (1998) & $10 \%$ & $10 \%$ \\
O VIII & Aichele et al. (1998) & $10 \%$ & $10 \%$ \\
Fe XXVI O'Rourke et al. (2001) & $15 \%$ & $15 \%$ \\
\hline
\end{tabular}

labeled Final error lists an estimate of error in the final cross section as compared to the experimental measurements. For example, if the the measurements are reproduced by the adopted cross sections to within the experimental error, then the experimental error is listed. If the adopted cross sections show deviations from the experimental measurements beyond the experimental errors, then an estimate of the typical deviation is listed.

\section{Ionization cross sections}

\subsection{The hydrogen isoelectronic sequence}

Aside from $\mathrm{HI}$, the available cross section measurements for the hydrogen isoelectronic sequence are listed in Table 1. For HI, the cross sections of Shah et al. (1987) have been selected as an accurate, representative set. FAC has been used to calculate the DI cross sections. In addition, there are the relativistic distorted wave cross sections of Fontes et al. (1999) who suggest that their parametric fits should be accurate for $Z \geq 4$ (Be IV).

For H I ionization cross section measurements cover more than 2 orders of magnitude in the incident electron energy. The BT scaled values are well fit and appear to approach the predicted high energy Bethe limit. For He II measurements are reported by Peart et al. (1969b), Dolder et al. (1961), Defrance et al. (1981a), and Achenbach et al. (1984). These values are all in agreement to within their quoted errors. The measurements of Peart et al. (1969b) extend to the highest energies and approach approach a value roughly 1.6 higher than the Bethe limit.

For the ions LiIII andB V, neither the FAC nor the Fontes et al. (1999) cross sections provide optimal fits to the data but the BT scaled cross section measurements are well described by 6 point spline fits.

For C VI, N VII and O VIII, the measurements are well described by the Fontes et al. (1999) cross sections. The C VI cross sections are shown in Fig. 2. The Fontes et al. (1999) cross sections are also in fairly good agreement with the measurements of O'Rourke et al. (2001), being about $15 \%$ below the measured values at low energies but in good agreement above about $2 I$.

To summarize, the final compilation of ionization cross sections uses the fits to the BT scaled measurements for the ions $\mathrm{HI}$, He II, Li III and B V. For Be IV the fits for B V are included with the appropriate ionization potential. For all other ions, the Fontes et al. (1999) cross sections are included.

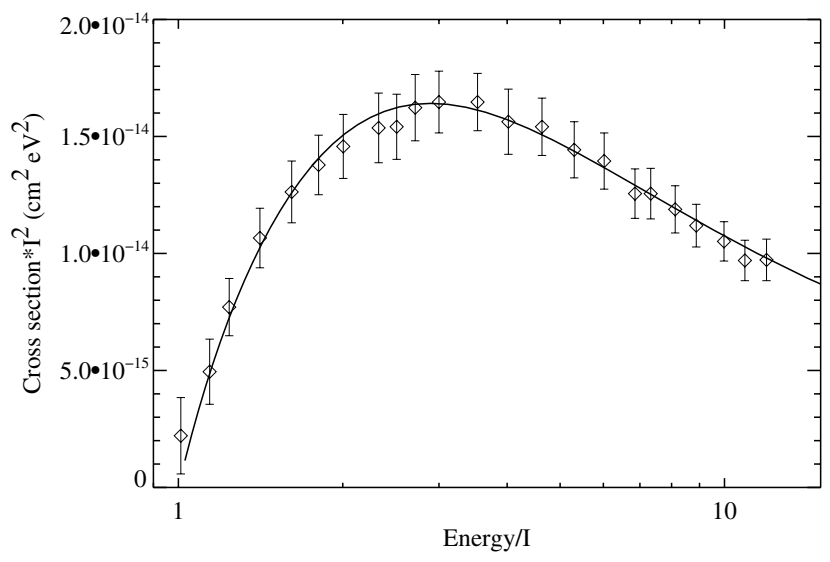

Fig. 2. Ionization cross sections for C VI. Diamonds - measurements of Aichele et al. (1998), solid line - calculations of Fontes et al. (1999).

\subsection{The helium isoelectronic sequence}

The available cross section measurements for the helium isoelectronic sequence are listed in Table 2. FAC has been used to calculate the DI cross sections. Fontes et al. (1999) has calculated relativistic distorted wave cross sections and provided parametric fits which are said to be accurate for $Z \geq 5$. At energies near $10 I$, they are generally higher that the FAC calculations since in the relativistic limit they approach a constant value rather than the classical non-relativistic Bethe limit.

For the ions $\mathrm{He}$ I and $\mathrm{Li}$ II, a distorted wave calculation can not be expected to accurately reproduce the measured cross sections and 6 point splines fit to the BT scaled measurements have been used. For B IV, the FAC cross sections are about $12 \%$ higher than the measurements at the peak of the cross section and the Fontes et al. (1999) cross sections do not accurately reproduce the measurements near threshold. Consequently, the BT scaled measurements were fit by a 6 point spline fit to provide greater accuracy. The FAC cross sections for C V reproduce the measurements of Crandall et al. (1979a) and Donets \& Ovsyannikov (1981) quite well while those of Bannister \& Havener (1996) show considerable cross section well below the threshold and are not considered further. The cross sections of Fontes et al. (1999) do not provide as good a fit. The FAC and measured cross sections for $\mathrm{C} \mathrm{V}$ are show in Fig. 3 The measurements for N VI are not in good agreement with the other measurements in the sequence. The measurements for Ne IX are reproduced by both the FAC cross sections and those of Fontes et al. (1999) to well within the experimental uncertainties.

For the final compilation of ionization cross sections, the fits to the BT scaled measurements for He I, Li II and B IV were used. For Be III, the fits for B IV were used with the appropriate ionization potential. For $\mathrm{C} \mathrm{V}$ through F VIII the FAC cross sections are used. For ions with higher $\mathrm{Z}$, the cross sections of Fontes et al. (1999) are included.

\subsection{The lithium isoelectronic sequence}

Measurements of ionization cross sections for ions in the lithium sequence are shown in Table 3. The experimental cross sections show a noticeable EA contribution for the more highly ionized species. DI and EA cross sections were calculated with FAC. The DI cross sections include ionization from the $1 \mathrm{~s}$ and $2 \mathrm{~s} l \mathrm{lev}-$ els. The EA cross sections include excitations to the $1 \mathrm{~s} 2 l 2 l$ ' and 1s2l3l' levels. Chen et al. (1996) has calculated EA ionization 
Table 2. Ionization cross section measurements for the helium isoelectronic sequence.

\begin{tabular}{|c|c|c|c|c|}
\hline Ion & Data source & Unused data source & $\begin{array}{l}\text { Experimental } \\
\text { error }\end{array}$ & $\begin{array}{l}\text { Final } \\
\text { error }\end{array}$ \\
\hline \multirow[t]{3}{*}{$\overline{\mathrm{He}} \mathrm{I}$} & Stephan et al. (1980) & & $8 \%$ & $10 \%$ \\
\hline & Montague et al. (1984b) & & $4 \%$ & \\
\hline & Shah et al. (1988) & & $6 \%$ & \\
\hline \multirow[t]{3}{*}{ Li II } & Lineberger et al. (1966) & & $12 \%$ & $20 \%$ \\
\hline & Peart et al. (1969b) & & $11 \%$ & \\
\hline & Wareing (1967) & & $15 \%$ & \\
\hline B IV & Crandall et al. (1979a) & & $4 \%$ & $10 \%$ \\
\hline \multirow[t]{2}{*}{$\mathrm{C} \mathrm{V}$} & Crandall et al. (1979a) & Bannister \& Havener (1996) & $7 \%$ & $10 \%$ \\
\hline & Donets \& Ovsyannikov (1981) & & $6 \%$ & \\
\hline N VI & Crandall et al. (1979a) & & $14 \%$ & $30 \%$ \\
\hline \multirow[t]{2}{*}{ Ne IX } & Donets \& Ovsyannikov (1981) & & $17 \%$ & $20 \%$ \\
\hline & Duponchelle et al. (1997) & & $11 \%$ & \\
\hline
\end{tabular}

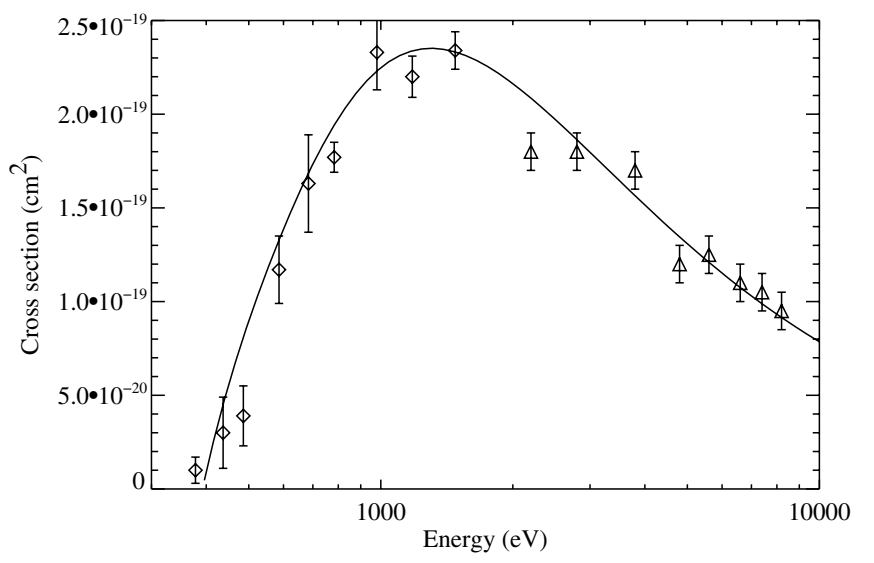

Fig. 3. FAC DI cross sections for $\mathrm{CV}$ and the measurements of Crandall et al. (1979a) (diamonds) and Donets \& Ovsyannikov (1981) (triangles, plotted with an arbitrary $10 \%$ experimental error).

rates for the lithium isoelectronic sequence and these agree well with the FAC calculations. The FAC cross sections are also in good agreement with the calculations of Reed \& Chen (1996) for N V and F VII, Chen \& Reed (1992) for Ar XVI and Fe XXIV and Badnell \& Pindzola (1993) for Fe XXIV.

For Li I, Jalin et al. (1973) and McFarland \& Kinney (1965) provide absolute measurements at high energies and Brink (1964) provides relative measurements at low energies. There is a difference of about a factor of 2.2 between the measurements of Jalin et al. (1973) and those of McFarland \& Kinney (1965). The measurements of Jalin et al. (1973) show the smoothest extension to the high energy limit and so this set is considered the most accurate. The measurements of McFarland \& Kinney (1965) were divided by a factor of 2.2 to provide a consistent set of measurements at high energy. The measurements of Brink (1964) were normalized to the high energy set and the BT scaled values of the 3 sets were fit with a 6-point spline fit. The error estimate refers to the high energy values.

For Be II and B III the measurements are well fit by a combination of a spline fit to the BT scaled DI cross sections and the addition of the FAC EA cross sections. In the case of C IV, the measurements of Teng et al. (2000) are about 22\% higher than those of Crandall et al. (1979b) and Howald \& Gregory (1984) but are in good agreement with the FAC DI and EA cross sections. The cross sections for N V, O VI and Ne VIII were well described by the FAC calculations although for Ne VIII the EA values appear to be somewhat underestimated. For the remaining members of the lithium isoelectronic sequence, the FAC values have been used. Cross sections over narrow ranges of energy near the EA peak have been measured by Kenntner et al. (1995) for Si XII and $\mathrm{Cl} \mathrm{XV}$. The estimated error in these measurements is $20 \%$ but the FAC cross sections are about a factor of 1.3 higher than the Kenntner et al. (1995) measurements. The FAC cross sections also agree with the measurements of Wong et al. (1993) for a single energy near the peak of the direct ionization cross section for the ions of TiXX, V XXI, CrXXII and MnXXIII and Fe XXIV. The FAC and experimental cross sections for $\mathrm{N} \mathrm{V}$ are shown in Fig. 4.

To summarize, the final compilation includes spline fits to the BT scaled measurements of Li I, spline fits to the DI cross sections plus the FAC EA cross sections for Be II and B III, and the FAC DI plus EA cross sections for all other ions.

\subsection{The beryllium isoelectronic sequence}

The measured cross sections for ions in the beryllium isoelectronic sequence are listed in Table 4. DI and EA ionization cross sections for the beryllium isoelectronic sequence have been calculated with FAC. The DI values include ionization from the $1 \mathrm{~s}$ and $2 \mathrm{~s}$ shells. The EA rates consider excitation to the $1 \mathrm{~s} 2 l^{3}$ and $1 \mathrm{~s} 2 l^{2} 3 l^{\prime}$ levels with the appropriate branching ratios. The FAC cross sections agree with the DI calculations of Younger (1981c) at the 10\% level. For Ne VII, the FAC values lie midway between the DI+EA cross sections of Chen et al. (1998) and Laghas et al. (1999). The FAC values also agree to with $10 \%$ with DI+EA values of Badnell \& Pindzola (1993) for Fe XXIII. From an inspection of Fig. 4 of Loch et al. (2003), the FAC cross sections also appear to be in good agreement with their calculations for $\mathrm{O} v$.

The BT scaled measurements for Be I and B II are well reproduced by a spline fit. It should be noted that Tawara (2002) provides cross sections for ionization of all neutrals considered in this study. However, the author provides no citation of where the values come from nor any estimates of the errors. These values are only used if no others seem to be available. Laboratory measurements are also available for C III, N IV and $\mathrm{OV}$ but these are apparently compromised by the existence of significant populations of ions in metastable $1 \mathrm{~s}^{2} 2 \mathrm{~s} 2 \mathrm{p}$ levels. This situation was previously noted by Arnaud \& Rothenflug (1985). Since their analysis, measurements of the ionization cross sections for Ne VII have been reported by Duponchelle et al. (1997) and Bannister (1996) that appear to be free of contamination by metastable levels. These measurements are well reproduced by the calculations of Laghas et al. (1999). The 
Table 3. Ionization cross section measurements for the lithium isoelectronic sequence.

\begin{tabular}{|c|c|c|c|c|}
\hline Ion & Data source & Unused data source & $\begin{array}{l}\text { Experimental } \\
\text { error }\end{array}$ & $\begin{array}{l}\text { Final } \\
\text { error }\end{array}$ \\
\hline \multirow[t]{3}{*}{ Li I } & Brink (1964) & & - & $15 \%$ \\
\hline & Jalin et al. (1973) & & $18 \%$ & \\
\hline & McFarland \& Kinney (1965) & & $10 \%$ & \\
\hline Be II & Falk \& Dunn (1983) & & $8 \%$ & $5 \%$ \\
\hline B III & Crandall et al. (1986) & & $7 \%$ & $5 \%$ \\
\hline CiV & Teng et al. (2000) & $\begin{array}{l}\text { Crandall et al. (1979b) } \\
\text { Howald \& Gregory (1984) }\end{array}$ & $14 \%$ & $12 \%$ \\
\hline $\mathrm{NV}$ & $\begin{array}{l}\text { Crandall et al. (1979b) } \\
\text { Defrance et al. (1990) }\end{array}$ & & $\begin{array}{l}7 \% \\
\geq 7 \%\end{array}$ & $15 \%$ \\
\hline O VI & $\begin{array}{l}\text { Crandall et al. (1979b) } \\
\text { Crandall et al. (1986) } \\
\text { Defrance et al. (1990) } \\
\text { Rinn et al. (1987) }\end{array}$ & & $\begin{array}{l}16 \% \\
7 \% \\
\geq 7 \% \\
8 \%\end{array}$ & $10 \%$ \\
\hline $\mathrm{Ne}$ VIII & $\begin{array}{l}\text { Defrance et al. (1990) } \\
\text { Aichele et al. (2000) } \\
\text { Donets \& Ovsyannikov (1981) } \\
\text { Duponchelle et al. (1997) } \\
\text { Riahi et al. (2001) }\end{array}$ & & $\begin{array}{l}\geq 8 \% \\
9 \% \\
7 \% \\
6 \% \\
-\end{array}$ & $15 \%$ \\
\hline Si XII & Kenntner et al. (1995) & & $20 \%$ & $30 \%$ \\
\hline $\mathrm{ClXV}$ & Kenntner et al. (1995) & & $20 \%$ & $30 \%$ \\
\hline Tixx & Wong et al. (1993) & & $11 \%$ & $11 \%$ \\
\hline V XXI & Wong et al. (1993) & & $10 \%$ & $10 \%$ \\
\hline Cr XXII & Wong et al. (1993) & & $9 \%$ & $9 \%$ \\
\hline Mn XXIII & Wong et al. (1993) & & $9 \%$ & $9 \%$ \\
\hline Fe XXIV & Wong et al. (1993) & & $9 \%$ & $9 \%$ \\
\hline
\end{tabular}

Table 4. Ionization cross section measurements for the beryllium isoelectronic sequence.

\begin{tabular}{lllll}
\hline \hline Ion & Data source & Unused data source & $\begin{array}{l}\text { Experimental } \\
\text { error }\end{array}$ & $\begin{array}{l}\text { Final } \\
\text { error }\end{array}$ \\
\hline Be I & Tawara (2002) & & - & $7 \%$ \\
B II & Falk et al. (1983) & $\begin{array}{l}\text { Falk et al. (1983) } \\
\text { C III }\end{array}$ & $\begin{array}{l}\text { Woodruff et al. (1978) } \\
\text { Falk et al. (1983) }\end{array}$ & \\
N IV & & $\begin{array}{l}\text { Howald \& Gregory (1984) } \\
\text { Crandall et al. (1979a) }\end{array}$ & \\
O v & & Falk et al. (1983) & & \\
& & & $9 \%$ & $6 \%$ \\
Ne VII & Bannister (1996) & & $6 \%$ & \\
& Duponchelle et al. (1997) & &
\end{tabular}

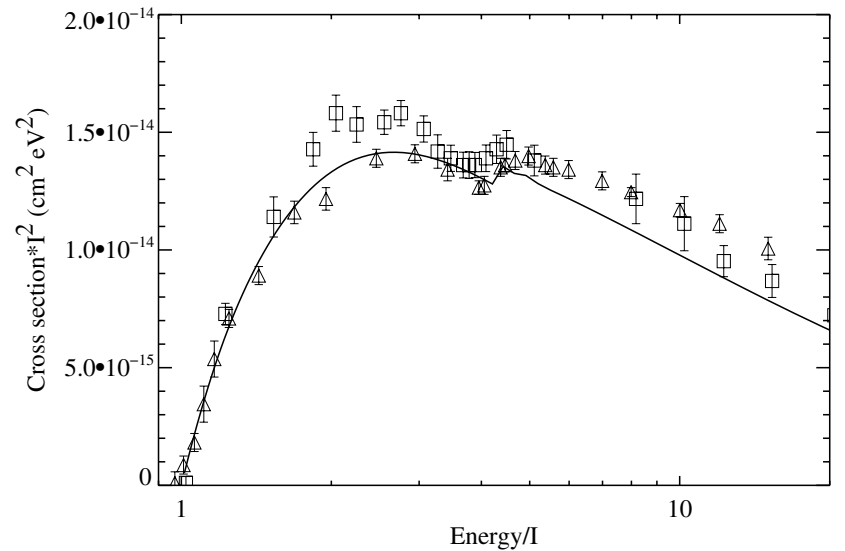

Fig. 4. FAC ionization cross sections of $\mathrm{NV}$ and experimental values. Triangles, Crandall et al. (1979a), squares, Defrance et al. (1990).

FAC cross sections and the measurements by Bannister (1996) and Duponchelle et al. (1997) for Ne VII are plotted together in
Fig. 5. It can be seen that the FAC cross sections reproduce the measurements quite well.

To summarize, the final compilation of ionization cross sections uses the spline fits to the BT scaled measurements for Be I and B II and the FAC cross sections for all of the remaining ions in the beryllium isoelectronic sequence.

\subsection{The boron isoelectronic sequence}

Table 5 shows the sources of cross section measurements for the boron isoelectronic sequence. There is no evidence for significant EA contributions to the measured cross sections. Consequently, FAC DI cross sections only, from the $n=1$ and $n=2$ shells, have been calculated for comparison with the measured values. The FAC values are in good agreement with the calculations of Chen et al. (1998) for Ne VI and Badnell \& Pindzola (1993) for Fe 22. From an inspection of Fig. 3 of Loch et al. (2003), the FAC cross sections also appear to be in good agreement with their calculations for O IV. 


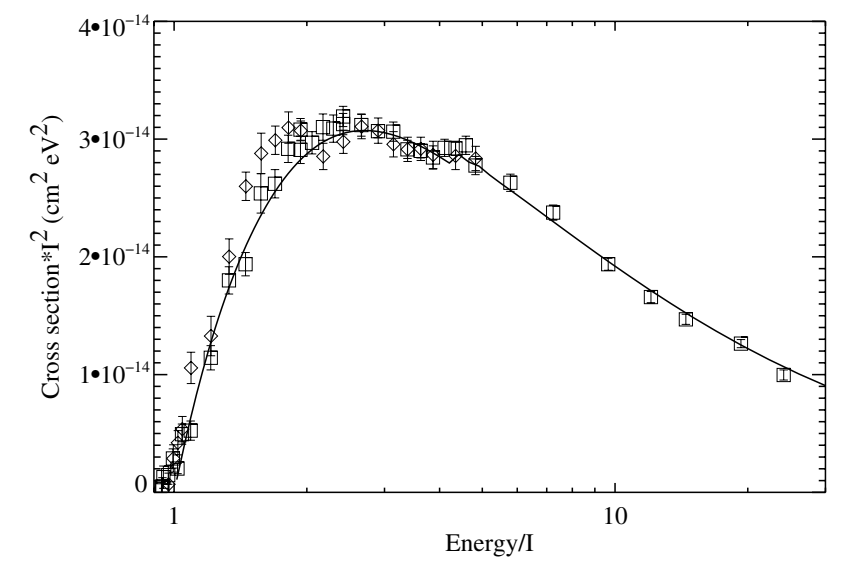

Fig. 5. Classically scaled ionization cross sections of Ne VII Diamonds - Bannister (1996), squares - Duponchelle et al. (1997), solid line - FAC.

Table 5. Ionization cross section measurements for the boron isoelectronic sequence.

\begin{tabular}{llll}
\hline \hline Ion & Data source & $\begin{array}{l}\text { Experimental } \\
\text { error }\end{array}$ & $\begin{array}{l}\text { Final } \\
\text { error }\end{array}$ \\
\hline B I & Tawara (2002) & - & \\
C II & Yamada et al. (1989a) & $10 \%$ & $10 \%$ \\
N III & Bannister \& Havener (1996) & $8 \%$ & $8 \%$ \\
& Mueller et al. (1983) & $9 \%$ & \\
O IV & Crandall et al. (1979a) & $8 \%$ & $8 \%$ \\
Ne VI & Bannister (1996) & $11 \%$ & $12 \%$ \\
& Duponchelle et al. (1997) & $6 \%$ & \\
\hline
\end{tabular}

For B I and C II, the measurements extend in energy to 1000 and 40 times threshold, respectively and fits to BT scaled measurements are satisfactory.

For the ions N III through Ne VI, the FAC cross sections underestimate the measured values from threshold to about twice threshold but are in good agreement at higher energies. For N III, two sets of measurements are available. The measurements of Mueller et al. (1985) extend from near threshold to about 5 times threshold while the measurements of Bannister \& Havener (1996) extend from near threshold to about 20 times threshold. The two are not in strict agreement but the average of the two is in better agreement with other measurements along the isoelectronic sequence than either set of measurements on their own. In order to better reproduce the low energy cross sections, fits to the BT scaled measurements of both sets of measurements have been used.

The only set of measurements for OIV are those of Crandall et al. (1979a). In the case of Ne VI, the measurements of Bannister (1996) Duponchelle et al. (1997) are in good agreement at energies below about 1.7 times threshold. Near the peak cross section, the measurements of Duponchelle et al. (1997) are about $20 \%$ higher than those of Bannister (1996). As there is no apparent reason to value one over the other, a fit to the combined measurements has been used.

To summarize, the final compilation of ionization rates uses spline fits to the BT scaled measurements for B I, C II, N III, O IV and Ne VI. For F V, a linear interpolations of the N III and Ne VI fit parameters is used with the appropriate ionization potential. For the ions Na VII through Zn XXVI, the FAC DI cross sections are used.

\subsection{The carbon isoelectronic sequence}

Measurements of ionization cross sections for the carbon isoelectronic sequence are listed in Table 6. There is no evidence of EA contributions to the cross sections and FAC has been used to calculate the DI values for ionization from the $n=1$ and $n=2$ shells. Very good agreement with the Fe XXI DI cross sections of Younger (1982a) is found. From an inspection of Fig. 2 of Loch et al. (2003), the FAC cross sections appear to be in good agreement with their calculations for O III. The FAC values also appear to agree with the calculations for Al VIII of Aichele et al. (2001) as shown in their Fig. 6.

The BT scaled measurements of C I were fit with a spline. There is reasonably good agreement $( \pm 30 \%)$ between the FAC calculations and the measurements for $\mathrm{N} \mathrm{II}, \mathrm{O}$ III and $\mathrm{Ne} \mathrm{V}$. However, fits to the BT scaled measurements provide a better reproduction of the measurements.

The measurements for Al VIII are described as problematical by the authors Aichele et al. (2001).

In summary, the final compilation of ionization cross sections used spline fits to the BT scaled measurements of C I, N II, $\mathrm{O}$ III and Ne V. For F IV, the O III and Ne V fits were linearly interpolated. For Al VIII and all other ions, the FAC DI cross sections are used.

\subsection{The nitrogen isoelectronic sequence}

Cross section measurements for the nitrogen isoelectronic sequence are listed in Table 7. There is no evidence for significant EA contributions and FAC has been used to calculate DI cross sections from the $n=1$ and $n=2$ shells. The FAC values are in good agreement with those of Chen et al. (1998) for Ne IV $( \pm 30 \%)$ and those of Younger (1982a) for Fe XX $( \pm 10 \%)$. For Ne IV, the measurements lie between between the two calculations. The FAC values also appear to agree with those of Aichele et al. (2001) for Al VII as shown in their Fig. 5.

Fits to the BT scaled cross sections for NI, O II, F III and Ne IV were satisfactory. The FAC cross sections for Ne IV agree well with the measurements with a maximum deviation of about $12 \%$ near the peak. Cross section measurements for Al VII are provided by Aichele et al. (2001). These appear to be enhanced by the presence of metastables in the beam and do not agree well with the FAC DI cross sections. The measured cross sections for Si VIII and Ar XII are in good agreement with the FAC DI cross sections.

To summarize, for the final compilation, fits to the BT scaled cross sections are used for N I through Ne IV and the FAC DI cross sections for the ions $\mathrm{Na} V$ through $\mathrm{Zn}$ XXIV.

\subsection{The oxygen isoelectronic sequence}

The available cross section measurements for the oxygen isoelectronic sequence are listed in Table 8 . As with the boron, carbon and nitrogen isoelectronic sequences, there is no evidence for significant EA contributions. FAC has been used to calculate DI cross sections from the $n=1$ and $n=2$ shells. The FAC cross sections are in good agreement $( \pm 10 \%)$ with those of Chen et al. (1998) for Ne III and those of Younger (1982a) for Fe XIX. The FAC values also appear to agree with those of Aichele et al. (2001) for Al VI as shown in their Fig. 4.

For OI, the measurements of Brook et al. (1978) and Thompson et al. (1995) were used. The cross sections of Brook et al. (1978) below $30 \mathrm{eV}$ indicated the presence of metastables so only their points at higher energies were included. 
Table 6. Ionization cross section measurements for the carbon isoelectronic sequence.

\begin{tabular}{lllll}
\hline \hline Ion & Data source & Unused data source & $\begin{array}{l}\text { Experimental } \\
\text { error }\end{array}$ & $\begin{array}{l}\text { Final } \\
\text { error }\end{array}$ \\
\hline C I & Brook et al. (1978) & $5 \%$ & $5 \%$ \\
N II & Yamada et al. (1989a) & $10 \%$ & $10 \%$ \\
O III & Falk et al. (1980) & $10 \%$ & $10 \%$ \\
Ne V & Bannister (1996) & $9 \%$ & $5 \%$ \\
& Duponchelle et al. (1997) & Aichele et al. (2001) & $5 \%$ & \\
Al VIII & & r & \\
\hline
\end{tabular}

Table 7. Ionization cross section measurements for the nitrogen isoelectronic sequence.

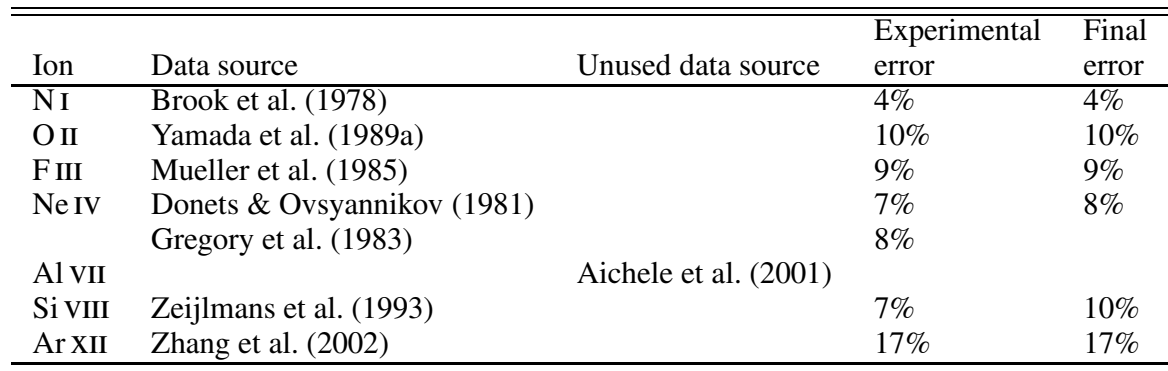

Table 8. Ionization cross section measurements for the oxygen isoelectronic sequence.

\begin{tabular}{|c|c|c|c|c|}
\hline Ion & Data source & Unused data source & $\begin{array}{l}\text { Experimental } \\
\text { error }\end{array}$ & $\begin{array}{l}\text { Final } \\
\text { error }\end{array}$ \\
\hline \multirow[t]{2}{*}{ O I } & Brook et al. (1978) & & $5 \%$ & $5 \%$ \\
\hline & Thompson et al. (1995) & & $5 \%$ & \\
\hline F II & Yamada et al. (1989b) & & $10 \%$ & $10 \%$ \\
\hline Ne III & Bannister (1996) & Matsumoto et al. (1990) & $9 \%$ & $9 \%$ \\
\hline $\mathrm{Al} \mathrm{VI}$ & & Aichele et al. (2001) & & \\
\hline Si VII & Zeijlmans et al. (1993) & & $7 \%$ & $12 \%$ \\
\hline $\operatorname{Ar} \mathrm{XI}$ & Zhang et al. (2002) & & $9 \%$ & $12 \%$ \\
\hline
\end{tabular}

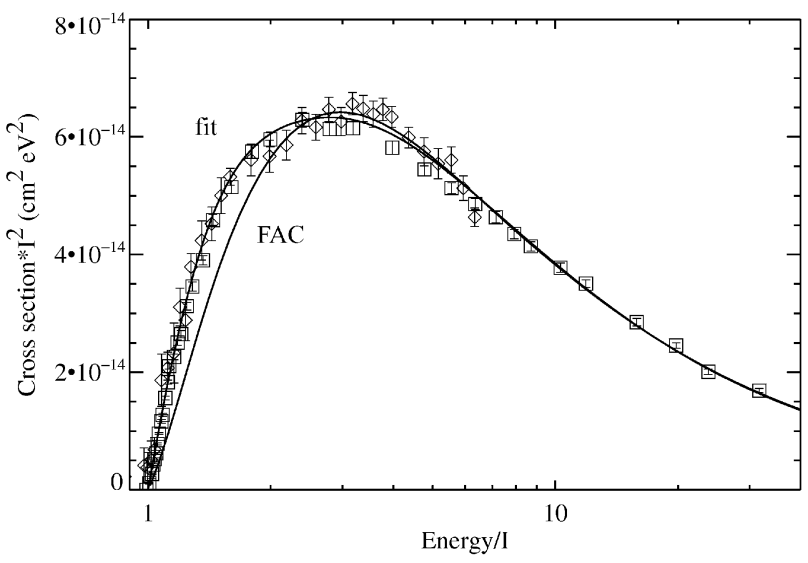

Fig. 6. Classically scaled ionization cross sections of Ne V. Diamonds Bannister (1996), squares - Duponchelle et al. (1997), solid lines FAC and the spline fit as noted.

Spline fits to the BT scaled cross sections for O I and F II provide a good reproduction of the measurements. For Ne III, the measurements of Bannister (1996) and Matsumoto et al. (1990) are in relatively good agreement, especially at energies less than roughly twice threshold and at energies above about 5 times threshold. At these energies, both are also in good agreement with the FAC DI cross sections. At the peak cross section, the values of Bannister are about $15 \%$ lower than those of Matsumoto et al. (1990). The FAC DI cross sections are essentially accurate to within experimental uncertainties. However, the fits to Bannister (1996) for the Ne III cross sections have been used.

For more highly ionized species in the oxygen isoelectronic sequence, measurements by Aichele et al. (2001) for Al VI, measurements of Zeijlmans et al. (1993) for Si VII, and measurements of Zhang et al. (2002) for Ar XI are available. For Al VI, Aichele et al. (2001) suggests that the enhanced cross section at threshold is due to a fraction of the beam ions in a metastable ${ }^{1} S_{0}$ state. Our FAC DI rates appear to be consistent with the DW rates calculated by Aichele et al. (2001). Zeijlmans et al. (1993) suggest that the Si VII measurements also indicate the presence of metastable ions. The measured cross sections for Si VII are roughly 10\% higher than the FAC cross sections. The Ar XI measurements are in good agreement with the FAC cross sections.

In summary, for the final compilation of ionization cross sections, spline fits to the BT scaled measurements are used for O I, F II and Ne III. Given the general level of agreement of the Ne III, Si VII and Ar XI measurements with the FAC DI cross sections, they are used for the ions Na IV through Zn XXIII.

\subsection{The fluorine isoelectronic sequence}

The available cross section measurements for the fluorine isoelectronic sequence are listed in Table 9. FAC has been used to 
Table 9. Ionization cross section measurements for the fluorine isoelectronic sequence.

\begin{tabular}{lllll}
\hline \hline Ion & Data source & Unused data source & $\begin{array}{l}\text { Experimental } \\
\text { error }\end{array}$ & $\begin{array}{l}\text { Final } \\
\text { error }\end{array}$ \\
\hline FI & Hayes et al. (1987) & & $20 \%$ & $20 \%$ \\
Ne II & Achenbach et al. (1984) & Müller et al. (1980) & $10 \%$ & $3 \%$ \\
& Diserens et al. (1984) & Yamada et al. (1989b) & $3 \%$ & \\
& Man et al. (1987b) & & $3 \%$ & \\
Al V & Aichele et al. (2001) & & $8 \%$ & $8 \%$ \\
Si VI & Thompson \& Gregory (1994) & $4 \%$ & $4 \%$ \\
ArX & Rachafi et al. (1987) & & $11 \%$ & $16 \%$ \\
\hline
\end{tabular}

Table 10. Ionization cross section measurements for the neon isoelectronic sequence.

\begin{tabular}{lllll}
\hline \hline & & & $\begin{array}{l}\text { Experimental } \\
\text { error }\end{array}$ & $\begin{array}{l}\text { Final } \\
\text { error }\end{array}$ \\
\hline Ion & Data source & Unused data source & $8 \%$ & $8 \%$ \\
& Almeida et al. (1995) & & $7 \%$ & \\
& Nagy et al. (1980) & $8 \%$ & \\
& Stephan et al. (1980) & $15 \%$ & \multirow{2}{*}{$6 \%$} \\
Na II & Wetzel et al. (1987) & Hirayama et al. (1986) & $13 \%$ & \\
& Hooper et al. (1966) & $10 \%$ & \\
Mg III & Peart \& Dolder (1968) & & $6 \%$ & \multirow{2}{*}{ Peart et al. (1969a) } \\
Al IV & & Aichele et al. (2001) & $8 \%$ & \\
Si v & & Thompson \& Gregory (1994) & & \\
Ar IX & Defrance et al. (1987) & & $10 \%$ & $10 \%$ \\
& Zhang et al. (2002) & & $5 \%$ & \\
\hline
\end{tabular}

calculate DI cross sections from the $n=1$ and $n=2$ shells. Calculations by Aichele et al. (2001) for Al V suggest that the EA contributions to the cross section can be disregarded. The FAC values are in good agreement $( \pm 12 \%)$ with the calculations of Laghas et al. (1999) for ArX and those of Younger (1982a) for Fe XVIII. The FAC values also appear to agree with those of Aichele et al. (2001) as shown in their Fig. 3.

For FI, the BT scaled measurements were well fit by a 6 point spline. For Ne II, the measurements of Achenbach et al. (1984), Diserens et al. (1984) and Man et al. (1987b) are in excellent agreement and extend to energies well above the threshold. The measurements of Yamada et al. (1989b) were in good agreement with these 3 sets of measurement but tended to diverge above $400 \mathrm{eV}$. Because the fit was well defined with the 3 sets of measurements just mentioned, the Yamada et al. (1989b) measurements were not included in the fits. The measurements of Müller et al. (1980) were at significant variance to all of the other sets and were not used. The BT scaled measurements of the 3 data sets are well fit with a 6 point spline.

The measurements of Al V Aichele et al. (2001) apparently correspond to excitation from the ground level alone and are in good agreement with the FAC and their CADW cross sections which include both direct and resonant ionization processes. The FAC cross sections for Si VI are in best agreement with the measured values of Thompson \& Gregory (1994) at low energies and are about $23 \%$ higher at higher energies. For Ar X, the measured cross sections Rachafi et al. (1987) are in good agreement with the FAC cross sections.

For a compilation of the complete set of cross sections for the fluorine isoelectronic sequence, fits to the measured rates for F I, $\mathrm{Ne}$ II, Al V and Si VI are used. For all other members of the sequence, the FAC DI rates are used.

\subsection{The neon isoelectronic sequence}

The available cross section measurements for the neon isoelectronic sequence are listed in Table 10. The FAC DI cross sections consider ionization from the $n=2$ shell only and are in good $( \pm 10 \%)$ agreement with the calculations of Younger (1981a) for Al IV, Ar IX and Fe XVII.

There are 4 sets of ionization cross sections measurements for Ne I. The measurements of Stephan et al. (1980) and Wetzel et al. (1987) are for energies less than $10 I$ and agree with each other. The measurements of Almeida et al. (1995) and Nagy et al. (1980) are for energies above $6 I$ and $20 I$ respectively and agree with each other as well. In the overlap region between 6 and 10 I there is a disagreement of about $13 \%$ which is outside the quoted errors. For Ne I a 6 point spline fit to all 4 sets of the BT scaled measurements is used.

For Na II, there are 3 sets of measurements which are in reasonable agreement except that the values of Hirayama et al. (1986) at energies above about $10 I$ do not tend toward the high energy limit as well as those of Peart \& Dolder (1968). Consequently, all of the data, except for the high energy points of Hirayama et al. (1986) has been used to arrive at a composite fit. The BT scaled measurements of Mg III byPeart et al. (1969a) are well described with a 6-point spline. Aichele et al. (2001) have analyzed their measurements for Al IV and suggest that the beam contained about about $20 \%$ of the ions in an excited state. When this is taken into account, the measurements seem to be consistent with the FAC direct ionization cross sections. Similarly, Thompson \& Gregory (1994) found that their measured cross sections for $\mathrm{Si} \mathrm{V}$ were affected by the presence of impurity metastable states. The two sets of Ar IX measurements were well fit by the FAC DI cross sections.

In summary, the fits to the BT scaled measurement for the Ne I, Na II and Mg III and the FAC DI cross sections for Al IV through Zn XXI are included in the final compilation. 
Table 11. Ionization cross section measurements for the sodium isoelectronic sequence.

\begin{tabular}{lllll}
\hline \hline Ion & Data source & Unused data source & $\begin{array}{l}\text { Experimental } \\
\text { error }\end{array}$ & $\begin{array}{l}\text { Final } \\
\text { error }\end{array}$ \\
\hline Na I & McFarland \& Kinney (1965) & Brink (1964) & $8 \%$ & $8 \%$ \\
& Zapesochnyi \& Aleksakhin (1969) & & $15 \%$ & \\
Mg II & Becker et al. (2004) & Crandall et al. (1982) & $10 \%$ & $10 \%$ \\
& Martin et al. (1968) & Müller et al. (1990) & $11 \%$ & \\
& Peart et al. (1991b) & & $9 \%$ & \\
Al III & Thomason \& Peart (1998) & Crandall et al. (1982) & $8 \%$ & $10 \%$ \\
Si IV & Crandall et al. (1982) & & $12 \%$ & $12 \%$ \\
Ar VIII & Rachafi et al. (1991) & Zhang et al. (1992) & $40 \%$ & $40 \%$ \\
Ti XII & Gregory et al. (1990) & & $7 \%$ & $8 \%$ \\
Cr XIV & Gregory et al. (1990) & & $10 \%$ & $25 \%$ \\
Fe XVI & Gregory et al. (1987) & & $14 \%$ & $20 \%$ \\
& Linkemann et al. (1995) & & $20 \%$ & \\
\hline
\end{tabular}

\subsection{The sodium isoelectronic sequence}

The available cross section measurements for the sodium isoelectronic sequence are listed in Table 11. FAC has been used to calculate the DI and EA cross sections. The DI cross sections include ionization from the $3 \mathrm{~s}$ and $n=2$ levels. The EA cross sections include excitation into the $2^{7} 3 l 3 l$ ' and $2^{7} 3 l 4 l$ ' levels.

For $\mathrm{NaI}$, the low energy range (1-6I)is covered by Zapesochnyi \& Aleksakhin (1969) and the high energy range $(20-100 I)$ is covered by McFarland \& Kinney (1965) and Brink (1964) which are in reasonable agreement. The measurements by McFarland \& Kinney (1965) show a more natural extrapolation to the high energy limit and so only the cross sections of Zapesochnyi \& Aleksakhin (1969) and McFarland \& Kinney (1965) have been used for the final fit. The BT-scaled cross sections for $\mathrm{Na}$ I data are well fit with a 7 point spline.

Five sets of measurements for Mg II are available. The measurements of Müller et al. (1990) were normalized to those of Crandall et al. (1982) by a multiplicative factor of 1.09 . The measurements of Crandall et al. (1982) are about $10 \%$ lower than those of Peart et al. (1991b) and Becker et al. (2004). The measurements of Martin et al. (1968) extend over a large energy range and agree with those of Peart et al. (1991b) and Becker et al. (2004) at low energies. There is no clear evidence for significant EA processes although resonant-excitationdouble-autoionization signatures are present. Consequently, the measurements of Martin et al. (1968), Peart et al. (1991b), and Becker et al. (2004) and been used and their BT-scaled Mg II data are well fit with a 6-point spline.

For Al III the FAC DI values are about $14 \%$ lower than those of Younger (1981b), agree well with the measurements of Thomason \& Peart (1998) and are about $30 \%$ higher than the measurements of Crandall et al. (1982). The Crandall et al. (1982) measurements have been normalized to those of Thomason \& Peart (1998) by a factor of 1.35 . The FAC EA cross sections, which are in agreement with those of Griffin et al. (1982a), are too large to explain the observed cross sections. This problem was noted by Griffin et al. (1982a) who suggested that the $2 p \rightarrow n p$ transitions were overestimated. It is not clear why these particular transitions should be problematic. For the results used here, the FAC EA cross sections have been multiplied by a factor of 0.4 to reproduce the measured values rather than excluding the $2 \mathrm{p} \rightarrow \mathrm{np}$ transitions.

For Si IV, the FAC DI cross sections are about $11 \%$ higher than those of Younger (1981b). The FAC EA cross sections are comparable to those, including the $2 p \rightarrow n p$ excitations, of
Griffin et al. (1982a), who noted that these were also larger than the measured values. The measurements are well described by the FAC DI cross sections plus the EA cross sections multiplied by a factor of 0.65 .

The measured cross sections of Ar VIII by Rachafi et al. (1991) and Zhang et al. (1992) do not agree very well. At low energies, the error bars are quite large. The FAC and Younger (1981b) DI cross sections agree at the $10 \%$ level. EA cross sections have been calculated by Tayal (1994), Reed \& Chen (1996), Teng \& Xu (1996) and Griffin et al. as reported by Zhang et al. (1992). The FAC DI + EA cross sections are in rough agreement with all of the above calculations except those of Griffin et al., which are approximately twice the value of the other calculations near the peak of the EA contribution at about $400 \mathrm{eV}$. In this area, their calculations agree with the measurements of Zhang et al. (1992). At energies above $700 \mathrm{eV}$, the FAC, Tayal (1994), Reed \& Chen (1996) and Teng \& Xu (1996) cross sections are in good agreement with the measurements of Rachafi et al. (1991) and the cross sections of Griffin et al. appear to approach these measurements as well. For Ar VIII, the FAC cross sections, without any scaling, provide a reasonable estimate of the ionization cross sections.

For Ti XII, the FAC cross sections are in good agreement with the measurements and the calculations of Griffin et al. (1987). For CrXIV, the FAC cross sections agree with the measurements and with the calculations of Griffin et al. (1987) and Qi et al. (2002) to within $20 \%$.

For FeXVI, the measurements measurements of Gregory et al. (1987) are about 30\% higher than those of Linkemann et al. (1995) near the peak of the EA contribution around $900 \mathrm{eV}$. The FAC cross sections lie between the two measurements at those energies and appear to agree with the calculations of Griffin et al. (1987) to with $10 \%$. The FAC cross sections for Ni XVIII agree with those of Pindzola et al. (1990) to better than $10 \%$.

In addition to DI and EA, resonant excitation double Auger (REDA) ionization LaGattuta \& Hahn (1981) contributes to ionization of ions in the sodium isoelectronic sequence. The first calculations of REDA cross sections were for Fe XVI by LaGattuta \& Hahn (1981). Later calculations by Griffin et al. (1987) suggested that the REDA process was not as important as originally reported by LaGattuta \& Hahn (1981), perhaps because of a simplification in their model. For this reason, and because of the difficulty in the calculation of REDA rates, they are not included in the present analysis.

To summarize, the final compilation of ionization cross sections includes the fits to the BT scaled measurements for $\mathrm{NaI}$ 
Table 12. Ionization cross section measurements for the magnesium isoelectronic sequence.

\begin{tabular}{lllll}
\hline \hline Ion & Data source & Unused data source & $\begin{array}{l}\text { Experimental } \\
\text { error }\end{array}$ & $\begin{array}{l}\text { Final } \\
\text { error }\end{array}$ \\
\hline Mg I & Boivin \& Srivastava (1998) & & $11 \%$ & $10 \%$ \\
& Freund et al. (1990) & & $10 \%$ & \\
& McCallion et al. (1992a) & & $12 \%$ & \\
Al II & Belic et al. (1987) & Montague et al. (1983) & $9 \%$ & $9 \%$ \\
Si III & Djurić et al. (1993b) & & $9 \%$ & $20 \%$ \\
S v & Howald et al. (1986) & & $9 \%$ & $20 \%$ \\
Cl vI & Howald et al. (1986) & $10 \%$ & $10-30 \%$ \\
Ar VII & Chung (1993) & Howald et al. (1986) & $6 \%$ & $10 \%$ \\
& & Zhang et al. (2002) & & \\
\hline
\end{tabular}

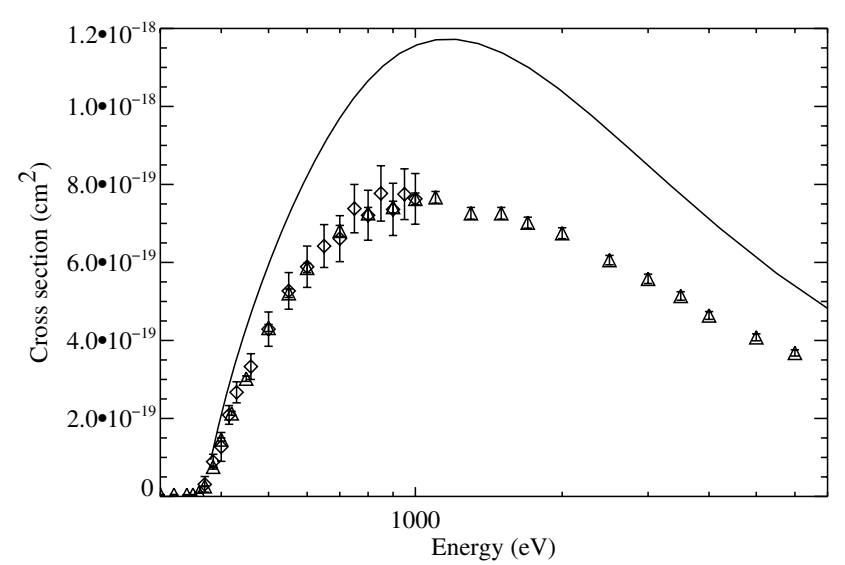

Fig. 7. Double ionization cross sections for ArVII. Triangles Tinschert et al. (1989b), squares - Zhang et al. (2002), solid line-FAC.

and $\mathrm{Mg}$ II, FAC cross sections with adjusted EA components for Al III and Si IV and unadjusted FAC cross sections for all other ions.

\subsection{The magnesium isoelectronic sequence}

The available cross section measurements for the magnesium isoelectronic sequence are listed in Table 12. Direct ionization from the $n=2$ shell results in an excited level that can autoionize into a neon-like ion, as previously suggested by Griffin \& Pindzola (1988). FAC has been used to calculate DI cross sections from the $n=2$ and $n=3$ shells. For DI from the $n=2$ shell, the branching ratios for radiative decay into a stable sodium-like ion relative to the autoionization to a neon-like ion have also been calculated and used to reduce the DI value. In all cases, autoionization leading to double ionization is the dominant process. The EA cross-sections for excitation into the $2 l^{7} 3 l^{3}$ and $2 l^{7} 3 \mathrm{~s}^{2} 4 l^{\prime}$ levels have been calculated with FAC. Theoretical distorted wave calculations of the ionization cross section of Si III (Djurić et al. 1993b), S V, Cl VI, and Ar VII (Pindzola et al. 1986a) and Fe XV (Mitnik et al. 1998) are in good agreement with the FAC values. Figure 7 shows measurements of double ionization in ArVII and the FAC direct ionization cross sections from the $n=2$ level corrected by the branching ratio. It appears that the measured cross section is about $66 \%$ of the FAC cross section, while the average calculated branching ratio for autoionization into Ar IX is about 0.93.

This leads to the question of which processes should be included in the determination of the ionization cross section for ions in the magnesium isoelectronic sequence. This question will become more apparent when the cross sections for $\mathrm{S} \mathrm{V}, \mathrm{Cl}$ VI,

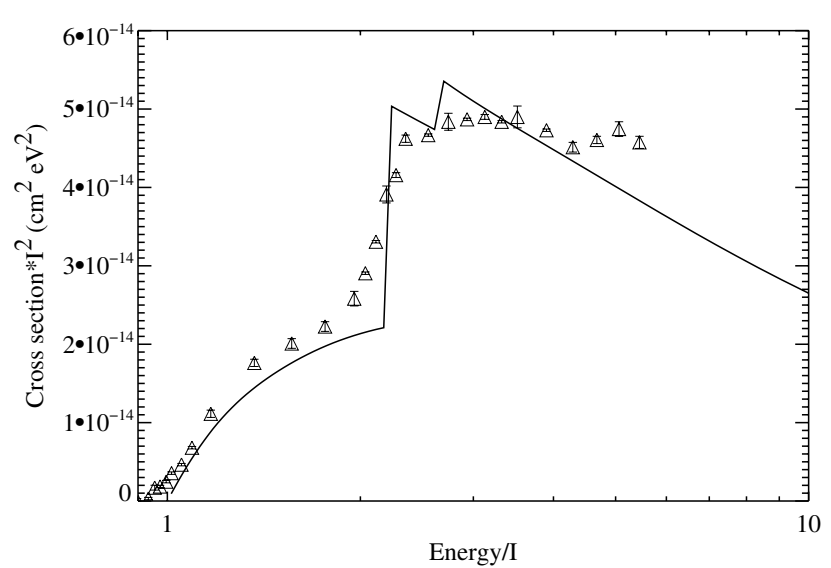

Fig. 8. Ionization cross sections for Ar VII. Measurements Chung (1993), solid lines - FAC.

and Ar VII are discussed below. Laboratory measurements typically refer to ionization into a particular ionization stage whether it is single, double, triple, etc. In order to maintain a consistent analysis, the cross sections and rate coefficients developed here pertain to single ionization processes.

For $\mathrm{MgI}$, the BT scaled measurements listed are well described by a 6 point spline fit. For Al II, the two sets of measurements agree well up to about 2.5 times threshold but those of Belic et al. (1987) showed the best extension to the high energy limit. The Belic et al. (1987) measurements suggest a small but insignificant contribution from EA processes. BT-scaled values of these measurements were fit with a 6 point spline.

Measurements are also available for the ions Si III, S v, Cl VI, and ArVII. A major uncertainty in these measurements is the presence of ions in $3 \mathrm{~s} 3 \mathrm{p}^{3} \mathrm{P}$ metastable levels. Their presence is clear in all of these measurements to varying degrees, except for those of ClVI and the measurements of Chung (1993) for Ar VII. These cross sections, shown in Fig. 8, are relatively well described by the FAC DI + EA cross sections.

To summarize our treatment of the $\mathrm{Mg}$ isoelectronic sequence, fits to the BT scaled measurements of $\mathrm{Mg}$ I and Al II have been used and for the remaining ions the FAC DI and EA cross sections are used.

\subsection{The aluminum isoelectronic sequence}

The available cross section measurements for the aluminum isoelectronic sequence are listed in Table 13. For all ions P III through Zn XVIII, the DI cross sections from the $n=2$ and $n=$ 3 shells have been calculated by FAC. As with the magnesium isoelectronic sequence, the DI cross section from the $n=2$ level 
Table 13. Ionization cross section measurements for the aluminum isoelectronic sequence.

\begin{tabular}{lllll}
\hline \hline Ion & Data source & Unused data source & $\begin{array}{l}\text { Experimental } \\
\text { error }\end{array}$ & $\begin{array}{l}\text { Final } \\
\text { error }\end{array}$ \\
\hline Al I & Freund et al. (1990) & & $10 \%$ & $10 \%$ \\
Si II & Djurić et al. (1993b) & $9 \%$ & $9 \%$ \\
Cl V & Bannister \& Guo (1993) & & $9 \%$ & $15 \%$ \\
Ar VI & Gregory \& Crandall (1982) & Mueller et al. (1983) & $11 \%$ & $15 \%$ \\
& & Zhang et al. (2002) & & \\
Fe XIV & Gregory et al. (1987) & & $16 \%$ & $20 \%$ \\
Ni XVI & Cherkani-Hassani et al. (2001) & & & $15 \%$ \\
\hline
\end{tabular}

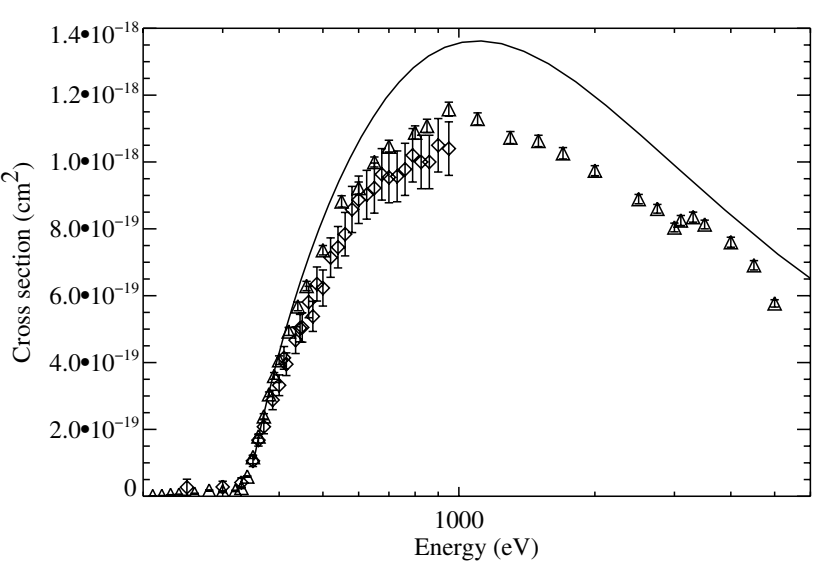

Fig. 9. Double ionization cross sections for Ar VI. Triangles Zhang et al. (2002), diamonds - Tinschert et al. (1989b), solid line FAC.

mostly results in double ionization. In Fig. 9 the measured and FAC values for the cross section for double ionization for ar VI are shown. The calculated values are roughly $20 \%$ higher than the measured values. For the ions P III through Ar VI FAC EA cross sections through the $2 \mathrm{p}^{6} 3 \mathrm{~s} 3 \mathrm{pn} l$ levels where $n=3,4,5$ are included. For P III through Zn XVIII the FAC EA cross sections through the $2 l^{7} 3 \mathrm{~s}^{2} 3 \mathrm{pn} l$ ' levels with $n=3,4$ are also included.

For Al I, the BT-scaled cross sections of Freund et al. (1990) are well reproduced by a 9 point spline fit. For Si II, Djuric et al. (1993b) pointed out the strong role of EA through the $2 \mathrm{p}^{6} 3 \mathrm{~s} 3 \mathrm{pn} l$ levels. These EA transitions are significantly greater than the DI cross sections at low energies. At higher energies, the EA cross section through the $2 \mathrm{p}^{5} 3 \mathrm{~s}^{2} 3 \mathrm{pn} l$ levels is also important Djurić et al. (1993b), and gains in importance for higher values of $\mathrm{Z}$. The FAC cross sections provide a fair reproduction of the Si II cross sections but a 9 point spline fit to the BT-scaled cross sections of Djurić et al. (1993b) is more accurate.

For $\mathrm{Cl} \mathrm{V}$ the measurements of Bannister \& Guo (1993) are well reproduced by the FAC calculations at low energies but at about $6 I$, the the FAC cross sections for single ionization are about $40 \%$ higher than the measured values. For Ar VI, all of the listed measurements are in reasonable agreement at energies above about $3 I$ and are well reproduced by the FAC cross sections. At lower energies, the measurements of Zhang et al. (2002) show evidence of significant metastable ion populations. At energies below $3 I$, the measurements of Gregory \& Crandall (1982) are well reproduced by the FAC cross sections. Figure 10 shows the classically scaled measurements and the FAC calculations for $\mathrm{Cl}$ V and Ar VI.

The Fe XIV cross section measurements of Gregory et al. (1987) are about $15 \%$ higher than the values calculated by FAC.

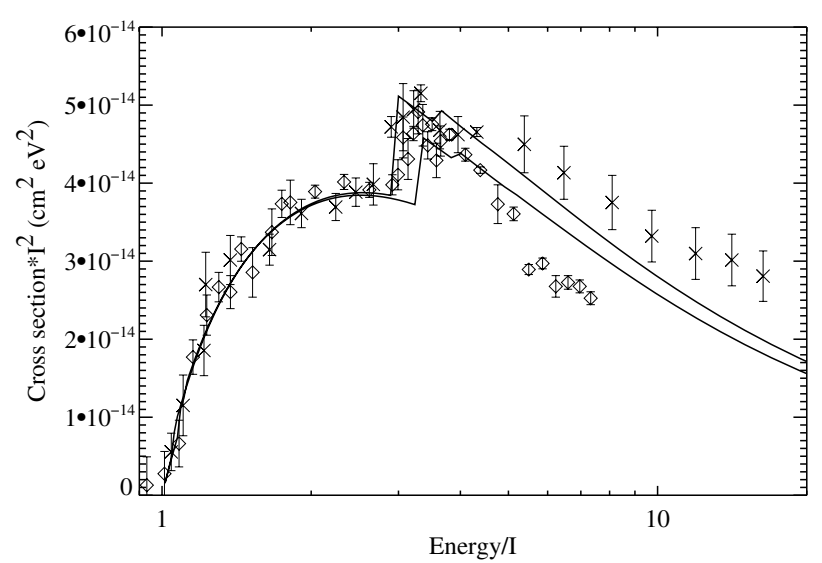

Fig. 10. Classically scaled ionization cross sections for $\mathrm{ClV}$ and Ar VI. Experimental points Bannister \& Guo (1993) (diamonds) and Gregory \& Crandall (1982) (crosses). FAC calculations for Cl V (bottom line) and Ar VI (top line).

The Ni XVI measurements of Cherkani-Hassani et al. (2001) are well described by the FAC values and also agree well with the calculations of Pindzola et al. (1990). The measurements of double ionization of Ni XVI by Cherkani-Hassani et al. (2001) agree well with the FAC double ionization cross sections from the $n=2$ level.

In summary, for the ions $\mathrm{AlI}$ and $\mathrm{Si}$ II the spline fits to the BT scaled measurements are included in the final compilation of ionization cross sections. For all other ions, the FAC DI and EA cross sections are used.

\subsection{The silicon isoelectronic sequence}

The available cross section measurements for the silicon isoelectronic sequence are listed in Table 14. DI cross sections from the $n=2$ (including the single ionization branching ratio) and $n=3$ levels have been calculated with FAC. EA cross sections have also been calculated with FAC and include excitations from the $n=3$ level into autoionizing levels of $n=3,4,5$ for the ions $\mathrm{S} \mathrm{III,} \mathrm{ClIV} \mathrm{and} \mathrm{ArV.} \mathrm{For} \mathrm{all} \mathrm{ions,} \mathrm{EA} \mathrm{cross} \mathrm{sections} \mathrm{from}$ the $n=2$ shell to the $n=3,4,5$ levels are included.

For Si I, the BT-scaled cross sections of Freund et al. (1990) are well reproduced by an 8 point spline fit. Similarly, for P II, the BT scaled cross sections of Yamada et al. (1989a) are well described by a 6 point spline fit.

The experimental S III cross sections of Yamada et al. (1988) are well matched by the FAC values near threshold and above 10 times threshold but exceed the measurements at intermediate energies by about $25 \%$. For ArV the experimental and FAC cross sections are shown in Fig. 11. The measurements of agree with each other to about $40 \%$ and there does not appear to 
Table 14. Ionization cross section measurements for the silicon isoelectronic sequence.

\begin{tabular}{|c|c|c|c|c|}
\hline Ion & Data source & Unused data source & $\begin{array}{l}\text { Experimental } \\
\text { error }\end{array}$ & $\begin{array}{l}\text { Final } \\
\text { error }\end{array}$ \\
\hline Si I & Freund et al. (1990) & & $10 \%$ & $10 \%$ \\
\hline P II & Yamada et al. (1989a) & & $10 \%$ & $10 \%$ \\
\hline S III & Yamada et al. (1988) & & $10 \%$ & $20 \%$ \\
\hline \multirow[t]{3}{*}{$\operatorname{Arv}$} & Crandall et al. (1979a) & & $6 \%$ & $30 \%$ \\
\hline & Müller et al. (1980) & & $10 \%$ & \\
\hline & Zhang et al. (2002) & & $4 \%$ & \\
\hline CrXI & Sataka et al. (1989) & & $5 \%$ & $15 \%$ \\
\hline \multirow[t]{2}{*}{ Ni XV } & & Wang et al. (1988) & & \\
\hline & & Cherkani-Hassani et al. (1999) & & \\
\hline
\end{tabular}

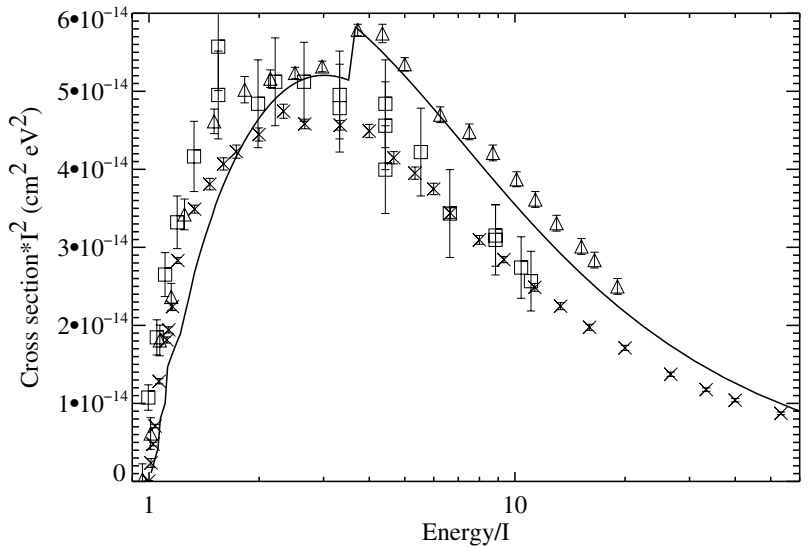

Fig. 11. Classically scaled ionization cross sections for ArV. Experimental points - Crandall et al. (1979a) (triangles), Müller et al. (1980) (squares), Zhang et al. (2002) (x), solid lines FAC.

be a logical way to decide on a consistent set of measurements. For Cr XI the measurement of Sataka et al. (1989) may indicate the presence of some ions in metastable levels in the beam but provide fair agreement with the FAC calculations, especially in the region where EA transitions are significant. For Ni XV, Griffin \& Pindzola (1988) showed that the measured rates of Wang et al. (1988) were commensurate with their calculations of ionization cross sections from the $3 \mathrm{p} 3 \mathrm{~d}$ excited configuration. There appears to be a similar problem with the measurements of Cherkani-Hassani et al. (1999) which do not agree well with those of Wang et al. (1988). Our FAC cross sections are in good agreement with the cross section of Griffin \& Pindzola (1988) from the ground level.

In summary, spline fits to the BT scaled cross sections of Si I and $\mathrm{P}$ II are included in the final compilation. There appears to be sufficient agreement between measured and the FAC cross sections for all ions S III through Z XVII that the FAC cross sections have been included as described.

\subsection{The phosphorus isoelectronic sequence}

The available cross section measurements for the phosphorus isoelectronic sequence are listed in Table 15. For Ar IV and all of the higher members of the phosphorus isoelectronic sequence, DI cross sections from the $n=2$ (adjusted for double ionization) and $n=3$ level has been calculated with FAC. For Ar IV it has been necessary to include EA cross sections from the $n=3$ level to the $n=3,4,5$ levels. For Ar IV and all of the higher members of the phosphorus isoelectronic sequence, EA cross sections from the $n=2$ shell to $n=4,5$ have been included.

For P I, the BT-scaled cross sections of Freund et al. (1990) are well reproduced by a 7 point spline fit. There are two sets of measurements for S II, those of Yamada et al. (1988) and those of Djurić et al. (1993a), and these are in agreement at about the $15 \%$ level. The cross sections of Yamada et al. (1988) are more numerous and extend to higher energy and they are used here. The BT scaled values are well reproduced by a 8 point spline fit. The $\mathrm{Cl}$ III are also well reproduced by a 7 point spline fit to the BT scaled measurements.

For ArIV, the measurements of Müller et al. (1980) and Gregory et al. (1983) show a significant cross section at the threshold suggesting that these measurements are compromised by metastables in the beam. The FAC cross sections are in rough agreement $( \pm 25 \%)$ with the measurements at higher energies.

For Fe XII the measurements of Gregory et al. (1987) indicate contributions to the DI cross section from metastable ions in the beam. At higher energies where $n=2$ EA contributions are important, their measurements are about $20 \%$ higher than the FAC cross sections which are in good agreement with the calculations of Pindzola et al. (1986b). For Ni XIV, our calculations are in very good agreement with the measurements of Cherkani-Hassani et al. (2001), see Fig. 12, and the calculations of Pindzola et al. (1990).

In summary, the fits to the BT scaled measurements of P I, $\mathrm{S}$ II and Cl III and the FAC cross sections, for all of the other ions, have been included in the final compilation of ions in the phosphorus isoelectronic sequence.

\subsection{The sulphur isoelectronic sequence}

The available cross section measurements for the sulphur isoelectronic sequence are listed in Table 16. For all ions from Ar III through $\mathrm{ZnXV}$ the FAC ionization cross section includes the DI cross section from the $n=2$ and $n=3$ levels and the FAC EA cross sections from the $n=2$ level to the $n=4,5$ levels. In addition, for Ar III through $\mathrm{Ca} V$, the FAC EA cross sections from the $n=3$ to the $n=3$ level are included.

For S I, the BT-scaled measurements of Freund et al. (1990) are well described by a 9-point spline fit. For Cl II, the measurements of Yamada et al. (1989b) are about 25\% higher than those of Djurić et al. (1993b) over most of their energy range, except near threshold where they are in good agreement. The measurements of Djurić et al. (1993b) are used because their classicallyscaled values are in better agreement with those of the Ar III. The BT-scaled Cl II measurements of Djurić et al. (1993b) are well described by a 6-point spline fit.

For ArIII, there are 6 sets of experimental measurements reported in the literature. The measurements by 
Table 15. Ionization cross section measurements for the phosphorus isoelectronic sequence.

\begin{tabular}{lllll}
\hline \hline Ion & Data source & Unused data source & $\begin{array}{l}\text { Experimental } \\
\text { error }\end{array}$ & $\begin{array}{l}\text { Final } \\
\text { error }\end{array}$ \\
\hline P I & Freund et al. (1990) & Djurić et al. (1993a) & $10 \%$ & $10 \%$ \\
S II & Yamada et al. (1988) & $5 \%$ & $15 \%$ \\
Cl III & Mueller et al. (1985) & Müller et al. (1980) & & $6 \%$ \\
Ar IV & & Gregory et al. (1983) & & \\
& & Gregory et al. (1987) & & \\
Fe XII & & & $8 \%$ & $10 \%$ \\
Ni XIV & Cherkani-Hassani et al. (2001) & &
\end{tabular}

Table 16. Ionization cross section measurements for the sulphur isoelectronic sequence.

\begin{tabular}{lllll}
\hline \hline & & & $\begin{array}{l}\text { Experimental } \\
\text { error }\end{array}$ & $\begin{array}{l}\text { Final } \\
\text { error }\end{array}$ \\
\hline Ion & Data source & Unused data source & $10 \%$ & $10 \%$ \\
Cl II & Djurić et al. (1990) & & $7 \%$ & $10 \%$ \\
Ar III & Danjo et al. (1983b) & Yamada et al. (1989a) & $10 \%$ & $20 \%$ \\
& Diserens et al. (1988) & & $3 \%$ & \\
& Man et al. (1993) & & $3 \%$ & \\
& Matsumoto et al. (1990) & & $10 \%$ & \\
& Müller et al. (1980) & & $10 \%$ & \\
Ti VII & Mueller et al. (1985) & Hartenfeller et al. (1998) & $9 \%$ & \\
Cr IX & & Sataka et al. (1989) & & \\
Fe XI & & Stenke et al. (1999) & & \\
Ni XIII & & Cherkani-Hassani et al. (1999) & & \\
& & Wang et al. (1988) & & \\
\hline
\end{tabular}

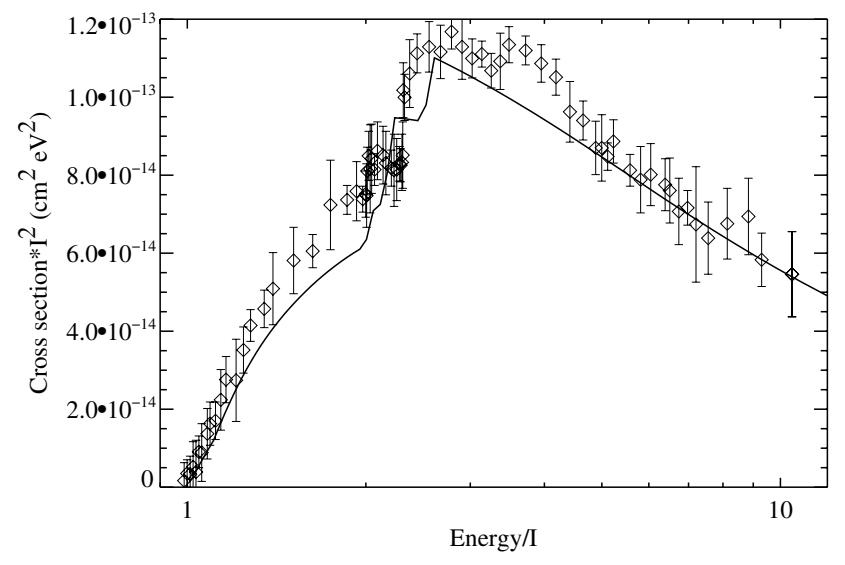

Fig. 12. Classically scaled values of Ni XIV cross sections. Points with error bars are the measurements of Cherkani-Hassani et al. (2001). Solid line is the FAC calculation.

Diserens et al. (1988) and Man et al. (1993) were made with the same apparatus and are nearly identical. The measurements by Danjo et al. (1984) and Matsumoto et al. (1990) are also nearly identical and appear to be by the same research group. The various measurements agree at the 10-25\% level. The FAC cross sections agree with these measurements at the $20 \%$ level.

For Ti VII, the measurements of Hartenfeller et al. (1998) were made on a beam containing both metastable and ground state ions. Their calculations are in reasonable agreement with the FAC calculations, and their measurements at high energies are also in reasonable agreement with the FAC calculations. The Sataka et al. (1989) measurements for Cr IX also show the presence of metastables in the beam and are generally about $25 \%$ higher than the FAC calculations. The Fe XI measurements also show strong indications of metastables and are about $35 \%$ higher than the FAC cross sections at high energies.

The experimental measurements for NiXIII by Cherkani-Hassani et al. (1999) and Wang et al. (1988) are in good agreement but pertain to ionization from an excited metastable state, based on a comparison with the calculations by Griffin \& Pindzola (1988). The FAC cross sections are in good agreement with those of Griffin \& Pindzola (1988) from the ground level.

In summary, the ionization rate coefficients are derived from the fits to the measured cross sections for S I and Cl II. For the other members of the sulphur isoelectronic sequence, the FAC ionization cross sections as described above are included in the final compilation.

\subsection{The chlorine isoelectronic sequence}

The available cross section measurements for the chlorine isoelectronic sequence are listed in Table 17. For the ions K III through Zn XIV, ionization cross sections have been calculated with FAC. These include the DI cross section from the $n=2$ (reduced by for double ionization) and $n=3$ shells and the EA cross sections from the $n=2$ level to the $n=4,5$ levels and from the $n=3$ to the $n=6,7,8$ levels.

For Cl I, the BT-scaled measurements of Hayes et al. (1987) are well described by a 9-point spline fit. For Ar II, there is good agreement among all of the measurements listed in Table 16 except for those of Müller et al. (1985a) which are significantly lower than the rest. The BT scaled cross sections of Ar II, excluding Müller et al. (1985a), are well fit with a 9-point spline.

For Ti VI, Hartenfeller et al. (1998) compared their measurements with those of Chantrenne et al. (1990) and distorted wave calculations. They find that the two sets of measurements are in good agreement but that they both lie between the ionization 
Table 17. Ionization cross section measurements for the chlorine isoelectronic sequence.

\begin{tabular}{lllll}
\hline \hline Ion & Data source & Unused data source & $\begin{array}{l}\text { Experimental } \\
\text { error }\end{array}$ & $\begin{array}{l}\text { Final } \\
\text { error }\end{array}$ \\
\hline Cl I & Hayes et al. (1987) & Müller et al. (1980) & $14 \%$ & $14 \%$ \\
Ar II & Gao et al. (1997) & & $3 \%$ & $5 \%$ \\
& Man et al. (1987b) & $3 \%$ & \\
& Müller et al. (1985a) & & $10 \%$ & \\
& Yamada et al. (1989b) & Chantrenne et al. (1990) & & \\
Ti VI & & Hartenfeller et al. (1998) & & \\
& & Sataka et al. (1989) & \\
Cr VIII & & Rejoub \& Phaneuf (2000) & & \\
Mn IX & & Gregory et al. (1987) & & \\
Fe X & & Stenke et al. (1995b) & $6 \%$ & $10 \%$ \\
Ni XII & Cherkani-Hassani et al. (1999) & & \\
\hline
\end{tabular}

Table 18. Ionization cross section measurements for the argon isoelectronic sequence.

\begin{tabular}{|c|c|c|c|c|}
\hline Ion & Data source & Unused data source & $\begin{array}{l}\text { Experimental } \\
\text { error }\end{array}$ & $\begin{array}{l}\text { Final } \\
\text { error }\end{array}$ \\
\hline \multirow[t]{6}{*}{ ArI } & Ma et al. (1991) & & $15 \%$ & $5 \%$ \\
\hline & McCallion et al. (1992b) & & $6 \%$ & \\
\hline & Nagy et al. (1980) & & $6 \%$ & \\
\hline & Stephan et al. (1980) & & $8 \%$ & \\
\hline & Straub et al. (1995) & & $3 \%$ & \\
\hline & Wetzel et al. (1987) & & $15 \%$ & \\
\hline \multirow[t]{3}{*}{ K II } & Hirayama et al. (1986) & & $13 \%$ & $6 \%$ \\
\hline & Hooper et al. (1966) & & $15 \%$ & \\
\hline & Peart \& Dolder (1968) & & $6 \%$ & \\
\hline Tiv & & Hartenfeller et al. (1998) & & \\
\hline Cr VII & & Sataka et al. (1989) & & \\
\hline Mn VIII & & Rejoub \& Phaneuf (2000) & & \\
\hline Ni XI & & Cherkani-Hassani et al. (1999) & & \\
\hline
\end{tabular}

rate they calculate from the ground state and from the $3 p^{4} 3 d$ excited state and concluded that there were significant levels of metastables in both beams. The FAC cross section sections are in generally good (15\%) agreement with the measurements at high energies.

Ionization cross sections of Cr VIII have been measured by Sataka et al. (1989). They found a significant cross section at energies below the ground level threshold and showed that these enhanced values were consistent with the direct ionization rate predicted by the Lotz Lotz (1967b) formula for ionization from a metastable $3 \mathrm{p}^{4} 3 \mathrm{~d}$ level. The FAC cross sections generally lie about $15 \%$ below the measurements of Sataka et al. (1989).

Cross sections for MnIX have been measured by Rejoub \& Phaneuf (2000). However, when the classically scaled measurements for ions in the chlorine isoelectronic sequence (Ti VI through Ni XII) are compared, the Mn IX values are the highest, for no apparent reason. Consequently the Mn IX measurements are not included.

Gregory et al. (1986) and Stenke et al. (1995b) have measured the ionization cross section of Fe X. Stenke et al. (1995b) shows there is good agreement between the two sets of measurements and that both are consistent with ionization from the $3 \mathrm{p}^{4} 3 \mathrm{~d}$ metastable level. The FAC cross sections lie about $25 \%$ below the measurements above about $900 \mathrm{eV}$ where the EA contributions are apparent and about $10 \%$ above the calculations by Pindzola et al. (1986b).

Cross sections for Ni XII have been measured by Cherkani-Hassani et al. (2001) and are in good agreement with the FAC calculations and those of Pindzola et al. (1990).
In summary, the fits to the BT scaled cross sections for $\mathrm{Cl}$ I and Ar II and the FAC cross sections, for all other ions in the $\mathrm{Cl}$ isoelectronic sequence, are included in the final compilation.

\subsection{The argon isoelectronic sequence}

The available cross section measurements for the chlorine isoelectronic sequence are listed in Table 18. Ionization cross sections have been calculated with FAC. These calculations included the DI cross section from the $n=3$ shell and the EA cross sections from the $n=2$ level to the $n=4,5$ levels and from the $n=3$ to the $n=4,5$ levels. Not all levels contribute to the EA transitions. For example, for V VI and above, most of the $n=4$ levels are below the ionization limit.

For ArI and K II, the measurements listed in Table 18 are well fit by a 6-point spline fit to the BT scaled values.

Measurements of the ions Ti V, Cr VII and Ni XI show strong evidence of significant levels of metastables in the beams. The measurements for Mn VIII show no evidence of a significant cross section below the ground level threshold. However, their classically-scaled values are higher than the other members of the sequence and also significantly higher than the FAC calculated values. For Ni XI, the FAC cross sections are in good agreement with those of Pindzola et al. (1990) from the ground level and in good agreement with the DI cross sections of Younger (1982b)

In summary, the fits to the BT scaled cross sections for ArI and K II are used for the final compilation of ionization cross sections. For the higher $\mathrm{Z}$ members of the argon isoelectronic sequence, there are no reliable ground state ionization 
Table 19. Ionization cross section measurements for the potassium isoelectronic sequence.

\begin{tabular}{lllll}
\hline \hline Ion & Data source & Unused data source & $\begin{array}{l}\text { Experimental } \\
\text { error }\end{array}$ & $\begin{array}{l}\text { Final } \\
\text { error }\end{array}$ \\
\hline K I & Brink (1964) & $20 \%$ & $15 \%$ \\
& Korchevoi \& Przonski (1967) & - & \\
& McFarland \& Kinney (1965) & - & \\
& Zapesochnyi \& Aleksakhin (1969) & $15 \%$ & $25 \%$ \\
Ca II & Peart \& Dolder (1975) & $10 \%$ & $8 \%$ \\
& Peart et al. (1989) & $8 \%$ & $9 \%$ \\
Sc III & Pindzola et al. (1994) & & $8 \%$ & \\
Ti IV & Falk et al. (1983) & van Zoest et al. (2004) & $9 \%$ & \\
Mn VII & & Rejoub \& Phaneuf (2000) & & \\
\hline
\end{tabular}

measurements. Consequently, the FAC cross section, based on their agreement with the calculations of Pindzola et al. (1990) for Ni XI, are included for all other ions in the argon isoelectronic sequence.

\subsection{The potassium isoelectronic sequence}

The available cross section measurements for the potassium isoelectronic sequence are listed in Table 19. FAC has been used to calculate the DI cross section from the $3 p$ and $3 d$ shells. The EA cross sections were calculated including excitation from the $3 \mathrm{p}^{6} 3 \mathrm{~d}$ to the $3 \mathrm{p}^{5} 3 \mathrm{~d} \mathrm{n} l$ levels with $n=4,5,6$.

For K I, the measurements are well fit by a 6-point spline fit to the BT-scaled values.

For CaII, the ground level is $3 \mathrm{p}^{6} 4 \mathrm{~s}$. Calculations by Burke et al. (1983), Griffin et al. (1984), Pindzola et al. (1987) and Badnell et al. (1991) have attempted to reproduce the ionization cross section of $\mathrm{Ca}$ II by including direct ionization and excitation-autoionization through the $3 \mathrm{p}^{5} 3 \mathrm{~d} 4 \mathrm{~s}$ and $3 \mathrm{p}^{5} 4 \mathrm{~s}^{2}$ levels. Badnell et al. (1991) has been the most successful in modeling the energy range between 25 and $45 \mathrm{eV}$, where the EA contributions are important, by means of an R-matrix calculation. FAC has been used to calculate the direct ionization cross sections from the $4 \mathrm{~s}$ and inner-shell $3 \mathrm{p}$ level and the EA cross sections through the the $3 p^{5} 3 d 4 s$ and $3 p^{5} 4 s^{2}$ levels. All of the calculations just mentioned required an adjustment to the DI cross sections below $25 \mathrm{eV}$ to reproduce the measured values and the current FAC DI cross section from the $4 \mathrm{~s}$ level has been multiplied by a factor of 0.5 . At energies above about $100 \mathrm{eV}$ there appears to be a significant contribution to the cross section from direct ionization from the $n=3$ level. With the modified DI cross sections plus the EA cross sections, a reasonable reproduction of the measured $\mathrm{Ca}$ II ionization cross sections are found although the Badnell et al. (1991) calculations provide a better fit in the region between 25 and $45 \mathrm{eV}$.

For Sc III the best reproduction of the measured cross sections has been with R-matrix calculations (Pindzola et al. 1994). For Sc III, the FAC cross sections are in good agreement with the measurements about about $2 I$ but not below that energy. The best reproduction of the measured cross sections are obtained with a 6-point spline fit to the BT scaled cross sections.

For TiIV the measurements of van Zoest et al. (2004) and Falk et al. (1983) are in reasonable agreement at high energies but differ by as much as $40 \%$ near the peak. Perhaps the most accurate calculations of the Ti IV cross section are the R-matrix results of Burke et al. (1984) and Gorczyca et al. (1994). These appear to agree somewhat better with the measurements of Falk et al. (1983) than those of van Zoest et al. (2004). The FAC distorted wave calculations for Ti IV significantly overestimate the measurements at energies somewhat above threshold but are in good agreement at high energies. Consequently, 7-point spline fits to the BT scaled cross sections of Falk et al. (1983) are used for Ti IV.

The measurements for Mn VII by Rejoub \& Phaneuf (2000) are about $80 \%$ higher than the FAC calculations. However, these measurements, when scaled classically, appear to be out of line with other measurements and calculations. In the case of $\mathrm{NiX}$, the FAC cross sections are about $30 \%$ lower than those of Pindzola et al. (1990).

To summarize, the fits to the BT scaled cross sections have been used for KI, Sc III and TiIV. For Ca II and the ions V V through Zn XII, the FAC cross sections have been used as described for the final compilation of ionization cross sections.

\subsection{The calcium isoelectronic sequence}

The available cross section measurements for the calcium isoelectronic sequence are listed in Table 20. For members of the calcium isoelectronic sequence, FAC has been used to calculate DI and EA cross sections. The DI cross sections include ionization from the $3 \mathrm{p}$ and $3 \mathrm{~d}$ shells. For the EA cross sections, autoionizing levels include the $n=3,4,5,6$ and 7 shells (Sc II through Mn VI), $n=4,5,6$ and 7 shells for all other ions.

For Ca I, the BT scaled values of the available measurements are well fit by a 9-point spline fit.

The Sc II measurements of Jacobi et al. (2004) are well described by their R-matrix calculations. In order for the FAC calculations to reproduce the measurements, it is necessary to multiply the FAC EA contribution by a factor of 0.4 . For Ti III, of the three sets of measurements those of Mueller et al. (1985) and Diserens et al. (1988) are in the best agreements. Those of Hartenfeller et al. (1998) indicate the presence of metastable ions in the beam and have not been included in the present analysis. The measurements are best reproduced if the FAC EA contributions are reduced by a factor of 0.4 . For Mn VI the measurements are in rough agreement with the FAC values. However, it should be noted that the measurements of Rejoub \& Phaneuf (2000) have often been problematical, as mentioned before. For Fe VII, the FAC cross section are in good agreement with the measured values and with the calculations of Pindzola et al. (1986b). The FAC cross sections for Ni IX are in fair agreement with the measurements but agree well (15\%) with the calculations of Pindzola et al. (1990).

For Ca I a spline fit to the BT scaled measured cross sections are used for the final compilation of ionization cross sections. For all other ions, the FAC calculations have been used. For Sc II and Ti III only, the FAC EA contributions have been reduced by a factor of 0.4 . 
Table 20. Ionization cross section measurements for the calcium isoelectronic sequence.

\begin{tabular}{|c|c|c|c|c|}
\hline Ion & Data source & Unused data source & $\begin{array}{l}\text { Experimental } \\
\text { error }\end{array}$ & $\begin{array}{l}\text { Final } \\
\text { error }\end{array}$ \\
\hline \multirow[t]{3}{*}{$\mathrm{Ca}$ I } & Okuno (1971) & & $8 \%$ & $8 \%$ \\
\hline & Rakhovskii \& Stepanov (1969) & & $24 \%$ & \\
\hline & Vainshtein et al. (1972) & & $16 \%$ & \\
\hline Sc II & Jacobi et al. (2004) & & $15 \%$ & $15 \%$ \\
\hline \multirow[t]{2}{*}{ Ti III } & Mueller et al. (1985) & Hartenfeller et al. (1998) & $9 \%$ & $25 \%$ \\
\hline & Diserens et al. (1988) & & $3 \%$ & \\
\hline Mn VI & Rejoub \& Phaneuf (2000) & & $25 \%$ & $50 \%$ \\
\hline \multirow[t]{2}{*}{ Fe VII } & Gregory et al. (1986) & & $5 \&$ & $12 \%$ \\
\hline & Stenke et al. (1999) & & $8 \%$ & \\
\hline Ni IX & Wang et al. (1988) & & $6 \%$ & $25 \%$ \\
\hline
\end{tabular}

Table 21. Ionization cross section measurements for the scandium isoelectronic sequence.

\begin{tabular}{llll}
\hline \hline Ion & Data source & $\begin{array}{l}\text { Experimental } \\
\text { error }\end{array}$ & $\begin{array}{l}\text { Final } \\
\text { error }\end{array}$ \\
\hline Sc I & Tawara (2002) & & \\
Ti II & Diserens et al. (1988) & $3 \%$ & $40 \%$ \\
& Hartenfeller et al. (1998) & $8 \%$ & \\
Fe VI & Gregory et al. (1986) & $7 \%$ & $10 \%$ \\
& Stenke et al. (1999) & $8 \%$ & \\
Ni VIII & Wang et al. (1988) & $6 \%$ & $15 \%$ \\
\hline
\end{tabular}

\subsection{The scandium isoelectronic sequence}

The available cross section measurements for the scandium isoelectronic sequence are listed in Table 21. For Ti II, which has a $3 d^{2} 4$ s ground configuration, FAC has been used to calculate the DI cross sections from the $4 \mathrm{~s}$ and $3 \mathrm{~d}$ shells and to calculate the EA cross sections for excitation to the $3 p^{5} 3 d^{3} 4 s$ level. For the remaining members of the scandium isoelectronic sequence, which have a $3 \mathrm{~d}^{3}$ ground configuration, FAC has been used to calculate the DI cross sections from the $3 p$ and $3 d$ levels and the EA cross sections from the $3 \mathrm{~s}$ and $3 \mathrm{p}$ levels to the $n=3,4,5,6$, and 7 levels. For Co VII through Zn X the $n=3$ levels are bound and do not contribute to EA.

For Sc I, the BT scaled measurements are well fit by by a 9 point spline, however, the experimental errors are unknown. The FAC cross sections for Ti II are in reasonably good agreement with the measurements, especially at the highest energies. The measurements for Fe VI are fit to $10 \%$ or better by the FAC cross sections which also appear to be in very good agreement with those of Pindzola et al. (1986b). The FAC cross sections for Ni VIII also agree with the measurements and the calculations of Pindzola et al. (1990) to better that $15 \%$.

Consequently, for ions in the scandium isoelectronic sequence, the spline fit to the BT scaled measurements of Sc I are used for the final compilation and the FAC DI and EA cross sections have been used as discussed above, for all other ions.

\subsection{The titanium isoelectronic sequence}

The available cross section measurements for the titanium isoelectronic sequence are listed in Table 22. FAC has been used to calculate the DI cross sections from the $3 p$ and $3 d$ shells and the EA cross sections, including excitation to the $n=3,4,5$, and 6 shells.

For Ti I, the BT scaled measurements of Tawara (2002) are well fit by by a 7 point spline. However, Tawara (2002) does not provide error estimates.
Table 22. Ionization cross section measurements for the titanium isoelectronic sequence.

\begin{tabular}{llll}
\hline \hline Ion & Data source & $\begin{array}{l}\text { Experimental } \\
\text { error }\end{array}$ & $\begin{array}{l}\text { Final } \\
\text { error }\end{array}$ \\
\hline Ti I & Tawara (2002) & & \\
Fe V & Stenke et al. (1999) & $8 \%$ & $8 \%$ \\
Ni VII & Wang et al. (1988) & $6 \%$ & $10 \%$ \\
\hline
\end{tabular}

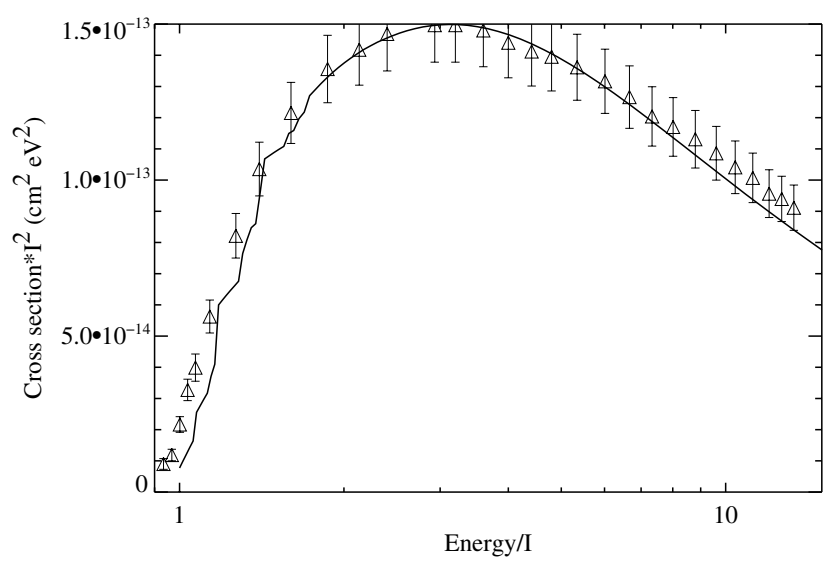

Fig. 13. Fe $\mathrm{V}$ ionization cross sections. Points with error bars are the measurements of Stenke et al. (1999). Solid line is the FAC cross section.

For Fe V, Stenke et al. (1999) were able to reproduce their measurements with their own distorted wave calculations. In the case of Ni VII, the measurements of Wang et al. (1988) are relatively well reproduced by the calculations of Griffin \& Pindzola (1988). The FAC cross sections for $\mathrm{Fe} \mathrm{V}$ are in good agreement with the measurements for $\mathrm{Fe} \mathrm{V}$ and for Ni VII. The Fe V cross sections are shown in Fig. 13.

For the final collection of ionization cross sections, the FAC calculations are used with the except of spline fits to the BT scaled measurements of Ti I.

\subsection{The vanadium isoelectronic sequence}

The available cross section measurements for the vanadium isoelectronic sequence are listed in Table 23. FAC has been used to calculate the DI cross sections from the $n=3$ level and the EA cross sections, including excitation from the $3 \mathrm{~s}$ and $3 \mathrm{p}$ levels to the $n=3$ shell and from the $3 p$ level to the $n=4$ shell.

For V I and Cr II, the BT scaled measurements are well fit by 6 point and 9 point splines, respectively. 
Table 23. Ionization cross section measurements for the vanadium isoelectronic sequence.

\begin{tabular}{|c|c|c|c|c|}
\hline Ion & Data source & Unused data source & $\begin{array}{l}\text { Experimental } \\
\text { error }\end{array}$ & $\begin{array}{l}\text { Final } \\
\text { error }\end{array}$ \\
\hline$\overline{V_{I}}$ & Tawara (2002) & & & \\
\hline Cr II & Man et al. (1987a) & \multirow{4}{*}{ Stenke et al. (1999) } & $2.5 \%$ & $5 \%$ \\
\hline Fe IV & & & & \\
\hline Ni VI & Wang et al. (1988) & & $6 \%$ & $15 \%$ \\
\hline & Stenke et al. (1995a) & & $6 \%$ & \\
\hline
\end{tabular}

For Fe IV, Stenke et al. (1999) notes that there seem to be a significant amount of metastable $\left(3 \mathrm{p}^{4} 4 \mathrm{~s}\right)$ impurity ions in their beam. The FAC cross sections are in good agreement with the CADW cross sections reported by Griffin et al. (1982b) and Stenke et al. (1999). For Ni VI the measurements of Wang et al. (1988) and Stenke et al. (1995a) are well described by the FAC calculations which are also in agreement with the calculations of Griffin \& Pindzola (1988).

For the final compilation of ionization cross sections, the fits to the BT scaled cross sections of V I and Cr II are used and the FAC cross sections are used for all other ions.

\subsection{The chromium isoelectronic sequence}

The available cross section measurements for the chromium isoelectronic sequence are listed in Table 24. The ground configuration of $\mathrm{Mn}$ II is $3 s^{2} 3 p^{6} 3 d^{5} 4 s$ and for more highly ionized members of the chromium isoelectronic sequence, it is $3 s^{2} 3 p^{6} 3 d^{6}$. For all ionized species, FAC has been used to calculate the DI and EA cross sections. For Mn II, DI cross sections from the $3 \mathrm{~d}$ and $4 \mathrm{~s}$ levels are included and EA cross sections for $3 p$ to $3 \mathrm{~d}$ and $n=4$ levels are included. For the other ions, DI from the $3 p$ and $3 \mathrm{~d}$ levels are included and EA excitations from the $3 \mathrm{~s}$ and $3 \mathrm{p}$ levels to the $3 \mathrm{~d}$ level and from the $3 \mathrm{p}$ levels to the $n=4$ shell.

For CrI there do not appear to be any available measurements of the ionization cross section. Tawara (2002) has apparently combined the theoretical values of Reid et al. (1992) at low energies and those of McGuire (1977) at high energies. The BT scaled values of Tawara (2002) are well fit by a 6 point spline.

For Fe III, the measurements of Mueller et al. (1985) and Stenke et al. (1999) were not used since they showed a significant cross section at the threshold indicating the presence of metastables in the beam. The FAC cross sections are somewhat larger than the CADW cross sections reported by Stenke et al. (1999), probably because more transitions have been included in the FAC calculations

The measurements for Ni V of Bannister \& Guo (1993) and Stenke et al. (1995a) are well-reproduced by the FAC calculations and are in very good agreement with the calculations of Pindzola et al. (1990).

In the final tabulation, FAC cross sections for all the members of the chromium isoelectronic sequence, aside from the spline fits to the BT scaled measurements of Cr I, have been included in the final compilation.

\subsection{The manganese isoelectronic sequence}

The available cross section measurements for the manganese isoelectronic sequence are listed in Table 25 . The ground configuration of Fe II is $3 s^{2} 3 p^{6} 3 d^{6} 4 s$ while for more highly ionized members of the chromium isoelectronic sequence, it is $3 \mathrm{~s}^{2} 3 \mathrm{p}^{6} 3 \mathrm{~d}^{7}$.
For Co III and above, FAC has been used to calculate the DI cross sections from the $3 p$ and $3 d$ levels.

For Mn I the BT scaled measurements are well fit by 6-point spline. In the case of Fe II and NiIV, the two sets of measurements by Stenke and colleagues show a significant cross section at threshold, indicated the presence of metastable ions. Consequently, 9 and 6 point spline fits to the BT scaled cross sections of Montague et al. (1984a) and Gregory \& Howald (1986), respectively, have been used.

The FAC DI cross sections for Ni IV overestimate the measurements by about $25 \%$ near the peak. The same situation applies to the cross sections of Pindzola et al. (1990). Since there is little structure in the cross section of Ni IV a spline fit to the scaled BT cross sections are a practical approach.

To summarize, the final compilation of ionization cross sections use the BT scaled fits for MnI, Fe II and Ni IV. The fits for Ni IV together with the appropriate ionization potential have been applied to the remaining members of the manganese isoelectronic sequence.

\subsection{The iron isoelectronic sequence}

The available cross section measurements for the iron isoelectronic sequence are listed in Table 26. FAC has been used to calculate the DI cross sections from the $3 \mathrm{~d}$ level.

For Fe I the BT scaled measurements are well fit by 6 point splines. It should be noted that the ionization cross sections of Tawara (2002) is an unreferenced compilation. In this case, the Fe I values appear to be a correction of Freund et al. (1990) which show a significant cross section at the ionization threshold, indicating the presence of metastable ions in their beam.

The measurements of Ni III by Stenke et al. (1995a) show a significant cross section at the threshold, indicating ions in metastable levels in the beam. However, at about $3 I$, their measurements agree with the CADW calculations of Pindzola et al. (1990). The FAC DI cross sections are 20-30\% higher than the values of Pindzola et al. (1990).

For $\mathrm{Cu}$ IV, the measurements are not well reproduced by the FAC DI cross sections and a 6 point spline fit to the BT scaled measurements is used.

The fits to the BT scaled cross sections of Cu IV have been used together with the appropriate ionization potential for all ions in the iron isoelectronic sequence aside from Fe I.

\subsection{The cobalt isoelectronic sequence}

The available cross section measurements for the cobalt isoelectronic sequence are listed in Table 27. For Co I, Ni II and Cu III, the BT scaled measurements are well fit by 6 point, 7 point and 6 point splines, respectively. For the remaining member of the cobalt isoelectronic sequence, the BT scaled fit for $\mathrm{Cu}$ III has been used together with the appropriate ionization potential.

\subsection{The nickel isoelectronic sequence}

For Ni I, the BT scaled measurements of Tawara (2002) are well fit by a 6 point spline. For $\mathrm{Cu}$ II and $\mathrm{Zn}$ III, there are no known measurements available and the FAC DI cross sections from the $3 \mathrm{~d}$ shell have been used. 
Table 24. Ionization cross section measurements for the chromium isoelectronic sequence.

\begin{tabular}{lllll}
\hline \hline Ion & Data source & Unused data source & $\begin{array}{l}\text { Experimental } \\
\text { error }\end{array}$ & $\begin{array}{l}\text { Final } \\
\text { error }\end{array}$ \\
\hline Cr I & Tawara (2002) & $\begin{array}{l}\text { Mueller et al. (1985) } \\
\text { Fe III }\end{array}$ & Stenke et al. (1999) & \\
Ni V & Bannister \& Guo (1993) & & $7 \%$ & $15 \%$ \\
& Stenke et al. (1995a) & & $8 \%$ & \\
\hline
\end{tabular}

Table 25. Ionization cross section measurements for the manganese isoelectronic sequence.

\begin{tabular}{lllll}
\hline \hline Ion & Data source & Unused data source & $\begin{array}{l}\text { Experimental } \\
\text { error }\end{array}$ & $\begin{array}{l}\text { Final } \\
\text { error }\end{array}$ \\
\hline Mn I & Tawara (2002) & & & \\
Fe II & Montague et al. (1984a) & Stenke et al. (1999) & $2.4 \%$ & $2.4 \%$ \\
Ni IV & Gregory \& Howald (1986) & Stenke et al. (1995a) & $5 \%$ & $5 \%$ \\
\hline
\end{tabular}

Table 26. Ionization cross section measurements for the iron isoelectronic sequence.

\begin{tabular}{lllll}
\hline \hline Ion & Data source & Unused data source & $\begin{array}{l}\text { Experimental } \\
\text { error }\end{array}$ & $\begin{array}{l}\text { Final } \\
\text { error }\end{array}$ \\
\hline Fe I & Tawara (2002) & Stenke et al. (1995a) & & \\
Ni III & & $4 \%$ & $8 \%$ \\
Cu IV & Gregory \& Howald (1986) & & $4 \%$ & 8 \\
\hline
\end{tabular}

Table 27. Ionization cross section measurements for the cobalt isoelectronic sequence.

\begin{tabular}{llll}
\hline \hline Ion & Data source & $\begin{array}{l}\text { Experimental } \\
\text { error }\end{array}$ & $\begin{array}{l}\text { Final } \\
\text { error }\end{array}$ \\
\hline Co I & Tawara (2002) & & \\
Ni II & Montague et al. (1984b) & $3 \%$ & $5 \%$ \\
Cu III & Gregory et al. (1986) & $4 \%$ & $4 \%$ \\
\hline
\end{tabular}

\subsection{The copper isoelectronic sequence}

The available cross section measurements for the copper isoelectronic sequence are listed in Table 28. For $\mathrm{Cu}$ I and $\mathrm{Zn} \mathrm{II}$, the BT scaled measurements are well fit by 6 point splines. The measurements of Freund et al. (1990) have not been used because of an apparent presence of metastable ions in their beam.

\subsection{The zinc isoelectronic sequence}

For $\mathrm{Zn}$ I the BT scaled measurements of Tawara (2002) are well fit by a 6 point spline.

\subsection{Error assessment}

Figure 14 shows a histogram of the final errors given in Tables 1 through 28. The median value of the final errors is $10 \%$ and the average value is $13 \%$. Generally, the final errors are smallest for the neutral and singly ionized species where the measurement are made with high accuracy. The cross sections are usually featureless and can be well fit. For highly ionized species, where the distorted wave FAC calculations should be the most accurate, there are typically few measurements and the accuracy of these measurements is not particularly high and so the final error is then also high.

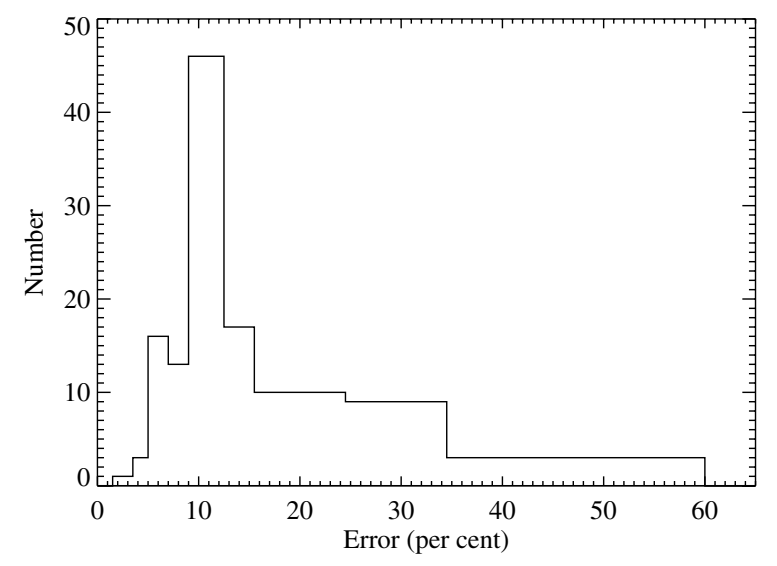

Fig. 14. Histogram of final errors.

\section{Ionization rate coefficients}

The ionization rate coefficients $R(T)$ for all ions of all elements hydrogen through zinc have been calculated by a 12 point Gauss-Laguerre integration over the ionization cross sections. The DI and EA rates were calculated separately and summed. A Burgess-Tully type scaling has been applied to these rates with a scaled temperature $x$,

$x=1-\frac{\log f}{\log (t+f)}$

where $t=k T / I$ and $k T$ is in the same units as the ionization potential $I$. A value of $f=2.0$ has been used for all fits presented here. The scaled rate coefficient $\rho$ is given by

$\rho=t^{\frac{1}{2}} I^{\frac{3}{2}} R(T) / E_{1}(1 / t)$

where $R(T)$ is the DI + EA ionization rate coefficient at the temperature $T$ and $E_{1}$ is the first exponential integral. 
Table 28. Ionization cross section measurements for the copper isoelectronic sequence.

\begin{tabular}{lllll}
\hline \hline Ion & Data source & Unused data source & $\begin{array}{l}\text { Experimental } \\
\text { error }\end{array}$ & $\begin{array}{l}\text { Final } \\
\text { error }\end{array}$ \\
\hline Cu I & Bolorizadeh et al. (1984) & Freund et al. (1990) & $13 \%$ & $13 \%$ \\
Zn II & Peart et al. (1991a) & & $10 \%$ & $10 \%$ \\
& Rogers et al. (1982) & $10 \%$ & \\
\hline
\end{tabular}

Table 29. Spline fits, multiplied by a factor of $10^{6}$, to the scaled ionization rate coefficients. Table 29 in its entirety is only available in electronic form at the CDS.

\begin{tabular}{llllllllllll}
\hline \hline $\begin{array}{l}Z \\
\text { Spline nodes ... } \\
\text { Spline values ... }\end{array}$ & NS & $I(\mathrm{eV})$ & $T_{\min }$ \\
\hline 2 & 1 & 20 & 24.587 & $1.000 \mathrm{e}+04$ & & & & & \\
0.0244 & 0.0500 & 0.1000 & 0.1500 & 0.2000 & 0.2500 & 0.3000 & 0.3500 & 0.4000 & 0.4500 & \\
& 0.5000 & 0.5500 & 0.6000 & 0.6500 & 0.7000 & 0.7500 & 0.8000 & 0.8500 & 0.9000 & 0.9151 \\
1.1152 & 1.2670 & 1.5608 & 1.8526 & 2.1431 & 2.4328 & 2.7222 & 3.0121 & 3.3048 & 3.6029 & \\
& 3.9066 & 4.2150 & 4.5277 & 4.8442 & 5.1551 & 5.4444 & 5.6808 & 5.7493 & 5.3837 & 5.1643 \\
2 & 2 & 20 & 54.418 & $2.239 \mathrm{e}+04$ & & & & & & \\
0.0247 & 0.0500 & 0.1000 & 0.1500 & 0.2000 & 0.2500 & 0.3000 & 0.3500 & 0.4000 & 0.4500 & \\
& 0.5000 & 0.5500 & 0.6000 & 0.6500 & 0.7000 & 0.7500 & 0.8000 & 0.8500 & 0.9000 & 0.9059 \\
1.6093 & 1.6839 & 1.8089 & 1.9142 & 2.0097 & 2.1001 & 2.1891 & 2.2779 & 2.3649 & 2.4510 & \\
& 2.5330 & 2.6077 & 2.6758 & 2.7302 & 2.7551 & 2.7353 & 2.6698 & 2.5085 & 2.1720 & 2.1219 \\
\hline
\end{tabular}

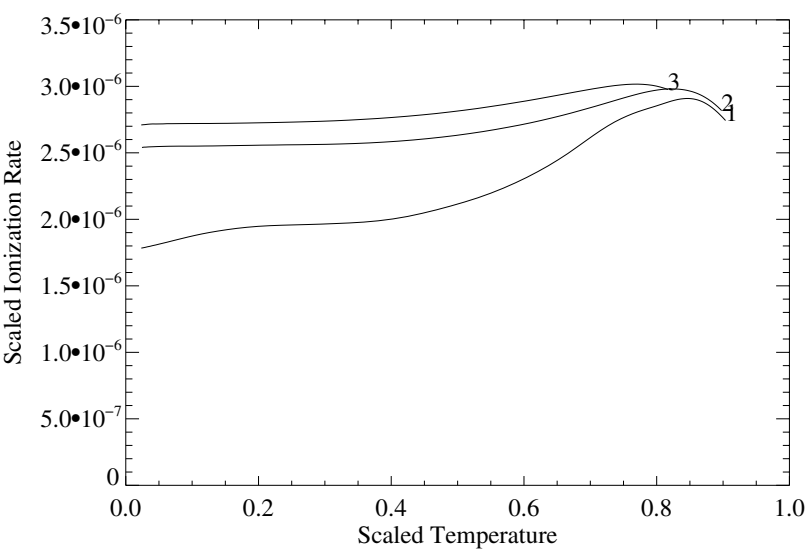

Fig. 15. BT scaled ionization rates for C IV (1), N V (2) and Fe XXIV (3).

Figure 15 shows BT scaled ionization rates for ions in the lithium isoelectronic sequence. It can be seen that the scaled rates are rapidly approaching the high $\mathrm{Z}$ limit.

Table 29 presents the spline fits to the BT scaled rate coefficients. The first line gives the nuclear charge $\mathrm{Z}$, the ionization stage (following spectroscopic notation), the ionization potential $I$ in $\mathrm{eV}$ and the minimum temperature for which the fits are applicable. The fits are accurate up to a temperature of $10^{9} \mathrm{~K}$ in all cases. The second entry, broken across two lines, are the values of $x$ where the spline has been fit. The third entry, also broken across two lines, are the values of the spline fit multiplied by $10^{6}$. The fact that the values of the spline fits are nearly constant indicates the usefulness of the scaling procedure. As the complete Table 29 is very large it is only available electronically and only the value of He I and He II are shown here.

To recover the rate coefficients from the scaled values, the inverse formulae are used:

$T=\left(\frac{I}{k}\right)\left(\exp \left[\frac{\log f}{1-x}\right]-f\right)$

$R=t^{-1 / 2} I^{-3 / 2} E_{1}(1 / t) \rho$.
As an example of how to apply Table 29 , for He II $(Z=2$, stage $=$ 2 ), a spline node value of $x=0.10$ corresponds to a temperature of $1.01 \times 10^{5} \mathrm{~K}$, using an ionization potential $I=54.42 \mathrm{eV}$ and $k=8.617 \times 10^{-5} \mathrm{eV} \mathrm{K}^{-1}$ in Eq. (6). The reduced temperature $t=$ $(k T / I)=0.16$. The spline value corresponding to this temperature is $1.8095 \times 10^{-6}$. The factor of $10^{-6}$ recovers the scaling used to create Table 29. Using Eq. (7), the ionization rate coefficient $R=3.06 \times 10^{-12} \mathrm{~cm}^{3} \mathrm{~s}^{-1}$. In order to determine the ionization rate coefficient at other temperatures, it is best to scale the temperature using Eq. (4) and then interpolate between the slowly varying scaled rate coefficients $\rho$.

In order to assess the differences between these new ionization rates and those of Arnaud \& Rothenflug (1985), Arnaud \& Raymond (1992), ionization equilibria have been calculated for the elements $\mathrm{C}, \mathrm{N}, \mathrm{O}, \mathrm{F}, \mathrm{Ne}, \mathrm{Mg}$, $\mathrm{Si}, \mathrm{Ca}, \mathrm{Fe}$, and $\mathrm{Ni}$ using the recombination rates of Mazzotta et al. (1998). These include the radiative recombination rates of Shull \& Steenberg (1982) and a compilation of dielectronic recombination rates from the literature. A comparison between these two sets of ionization equilibrium shows typical differences ranging from negligible to 20-30\%. The greatest differences were found for the ions Ni V through Ni XI that can be traced to the fact that Arnaud \& Rothenflug (1985) did not include EA rates for ions with more than 18 electrons (beyond the Ar isoelectronic sequence). For the case of iron, this was corrected by Arnaud \& Raymond (1992). For some of these nickel ions, differences can approach $60 \%$. Similar problems are likely to be found for the elements cobalt, copper and zinc but these do not have very high astrophysical abundances. Another significant difference is for the ions $\mathrm{C} \mathrm{V}$ and C VI and is attributable to an apparent error in ionization equilibrium tabulation by Mazzotta et al. (1998) for these ions and not their stated ionization rate. This error is apparently widely recognized.

In Fig. 16, the ionization rate coefficients for the ions Fe IX, Fe XI,Fe XIII, and Fe XV developed here are plotted together with the rates from Arnaud \& Raymond (1992). The differences can be as large as about $30 \%$. Similarly, the present rate coefficients for the ions Ni V, Ni VII, and Ni IX are shown in Fig. 17 together 


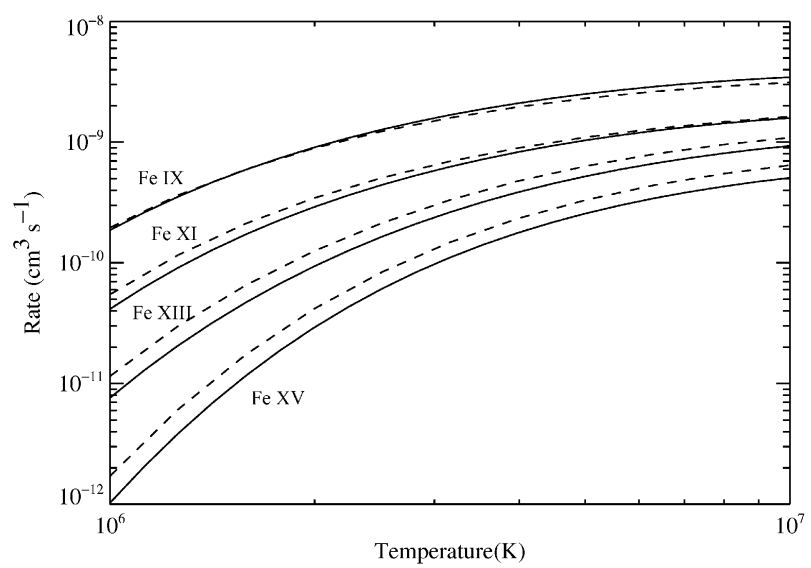

Fig. 16. Ionization rate coefficients for selected iron ions. Solid line present results, dashed line - Arnaud \& Raymond (1992).

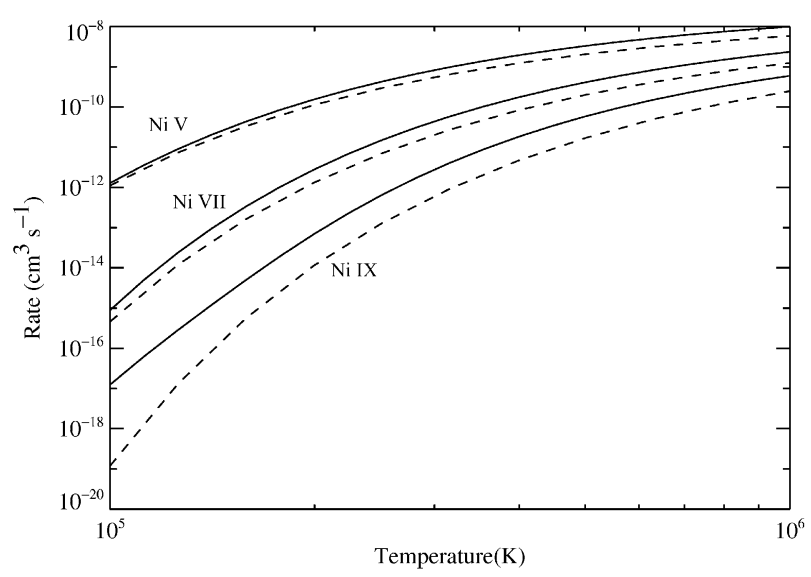

Fig. 17. Ionization rate coefficients for selected nickel ions. Solid line present results, dashed line - Arnaud \& Rothenflug (1985).

with those of Arnaud \& Rothenflug (1985). The differences can be quite substantial and would affect the calculated ionization equilibria.

\section{Discussion}

Through the use of a combination of ionization scaling laws and atomic calculations with FAC and other codes, it has been possible to reproduce measured electron ionization cross section to a fair degree of accuracy, generally on the order of about $20 \%$ or better. In all cases, the high-energy Bethe limit for the ionization cross sections has been incorporated.

Since the earlier investigations of electron ionization rates of Arnaud \& Rothenflug (1985), Arnaud \& Raymond (1992), there have been a considerable number of reported measurements of ionization cross sections. Future improvements in our knowledge of ionization cross sections will likely come about through improvements in our ability to calculate these cross sections and through new measurements. Further, the current set of cross sections and rate coefficients refer to ionization from the ground level. The inclusion of ionization from metastable levels will be useful in exploring the ionization state of plasmas, such as the solar corona, where metastable levels have significant populations, e.g. Nussbaumer \& Storey (1975).

\section{Summary}

A complete, new set of ground level electron collision ionization rate coefficients has been developed for all atoms and ions of the elements from hydrogen through zinc. These are based on an analysis of measurements of ionization cross sections and theoretical calculations with FAC and other codes. The use of scaling laws for ionization has facilitated this analysis. Differences between these rates and those of Arnaud \& Rothenflug (1985) and Arnaud \& Raymond (1992) are not particularly large except for some ions of nickel. Fits to the scaled rate coefficients are presented here as an electronically available table. In the near future, the cross sections and rate coefficients will be inserted into the CHIANTI atomic database where they will be freely available.

Acknowledgements. I gratefully acknowledge the National Institute for Fusion Science (NIFS) which maintains the AMDIS database (https://dbshino.nifs.ac.jp) from which a very large fraction of the measured and theoretical ionization cross sections used in this analysis were taken. This research has made extensive use of NASA's Astrophysics Data System. This work has depended heavily on the freely-available Flexible Atomic Code (FAC) developed by M. F. Gu. I would also like to thank S. Cherkani-Hassan and P. DeFrance for providing ionization cross section data in digital form. All of our work on the CHIANTI database makes continued use of the National Institute of Standards and Technology (NIST) Atomic Spectra Database (http://physics.nist.gov/PhysRefData/ASD). I would also like to thank Ms. Stephanie Evans for her help in the early stages of this project. This work has been supported by NASA's Living with a Star Targeted Research and Technology program and by the Solar and Heliospheric Physics Supporting Research and Technology program.

\section{References}

Achenbach, C., Müller, A., Salzborn, E., \& Becker, R. 1984, J. Phys. B, 17, 1405 Aichele, K., Hartenfeller, U., Hathiramani, D., et al. 1998, J. Phys. B, 31, 2369 Aichele, K., Shi, W., Scheuermann, F., et al. 2000, Phys. Rev. A, 63, 014701 Aichele, K., Steidl, M., Hartenfeller, U., et al. 2001, J. Phys. B, 34, 4113 Almeida, D. P., Fontes, A. C., \& Godinho, C. F. L. 1995, J. Phys. B, 28, 3335 Arnaud, M., \& Raymond, J. 1992, ApJ, 398, 394 Arnaud, M., \& Rothenflug, R. 1985, A\&AS, 60, 425 Badnell, N. R., \& Pindzola, M. S. 1993, Phys. Rev. A, 47, 2937

Badnell, N. R., Griffin, D. C., \& Pindzola, M. S. 1991, J. Phys. B, 24, L275 Bannister, M. E., \& Guo, X. Q. 1993, unpublished, http://www-cfadc.phy.ornl.gov/xbeam/xbmintro.html Bannister, M. E., \& Havener, C. C. 1995, unpublished, http://www-cfadc . phy.ornl.gov/xbeam/xbmintro.html Bannister, M. E. 1996, Phys. Rev. A, 54, 1435

Becker, C., Knopp, H., Jacobi, J., et al. 2004, J. Phys. B., 37, 1503

Belic, D. S., Falk, R. A., Timmer, C., \& Dunn, G. H. 1987, Phys. Rev. A, 36, 1073

Bethe, H. 1930, Ann. Phys., 5, 325

Biemont, E., Fremat, Y., \& Quinet, P. 1999, ADNDT, 71, 117

Boiko, V. A., Pal'chikov, V. G., Skobelev, I. Yu., \& Faenov, A. Ya. 1988, Atomic spectroscopical constants., Moscow, Standarts, 1, http://spectr-w3.snz.ru/index.phtml

Boivin, R. G., \& Srivastava, S. K. 1998, J. Phys. B, 31, 2381

Bolorizadeh, M. A., Patton, C. J., Shah, M. B., \& Gilbody, H. B. 1994, J. Phys. $\mathrm{B}, 27,175$

Bottcher, C., Griffin, D. C., \& Pindzola, M. S. 1983, J. Phys. B, 16 L65

Brink, G. O. 1964, Phys. Rev., 134, A345

Brook, E., Harrison, M. F. A., \& Smith, A. C. H. 1978, J. Phys. B, 11, 3115

Burgess, A., \& Tully, J. A. 1992, A\&A, 254, 436

Burke, P. G., Kingston, A. E., \& Thompson, A. 1983, J. Phys. B, 16, L385

Burke, P. G., Fon, W. C., \& Kingston, A. E. 2004, J. Phys. B, 17, L773

Chantrenne, S. J., Gregory, D. C., Buie, M. J., \& Pindzola, M. S. 1990, Phys. Rev. A, 41, 140

Chen, M. H., \& Reed, K. J. 1992, Phys. Rev. A, 45, 4525

Chen, C., Hu, W., Fang, D., Wang, Y., \& Yang, F. 1996, ADNDT, 64, 301

Chen, C., Yan, S., Teng, Z., et al. 1998, J. Phys. B, 31, 2667

Cherkani-Hassani, S., Defrance, P., \& Oualim, E. M. 1999, Phys. Scr., T80, 292

Cherkani-Hassani, S., Kouilid, S. M., \& Defrance, P. 2001, Phys. Scr., T92, 287

Chung, Y.-S. 1993, unpublished, http://www-cfadc.phy.ornl.gov/xbeam/xbmintro.html 
Crandall, D. H., Phaneuf, R. A., \& Gregory, D. C. 1979a, Electron Impact Ionization of Multicharged Ions, ORNL/TM-7020, Oak Ridge National Laboratory,

http://www-cfadc.phy . ornl.gov/xbeam/xbmintro.html

Crandall, D. H., Phaneuf, R. A., Hasselquist, B. E., \& Gregory, D. C. 1979b, J. Phys. B, 12, L249

Crandall, D. H., Phaneuf, R. A., Falk, R. A., Bélic, D. S., \& Dunn, G. H. 1982, Phys. Rev. A, 25, 143

Crandall, D. H., Phaneuf, R. A., Gregory, D. C., et al. 1986, Phys. Rev. A, 34, 1757

Danjo, A., Matsumoto, A., Ohtani, S., et al. 1984, J. Phys. Soc. Jpn, 53, 4091

Defrance, P., Brouillard, F., Claeys, W., Cornet, A., \& Van Wassenhove, G. 1981a, J. Phys. B, 14, 103

Defrance, P., Claeys, W., Cornet, A., \& Poulaert, G. 1981b, J. Phys. B, 14, 111

Defrance, P., Rachafi, S., Jureta, J., Meyer, F., \& Chantrenne, S. 1987, Nucl. Instr. Meth. B, 23, 265

Defrance, P., Chantrenne, S., Rachafi, S., et al. 1990, J. Phys. B, 23, 2333

Dere, K. P., Landi, E., Mason, H. E., Monsignori Fossi, B. C., \& Young, P. R. 1997, A\&ASS, 125, 149

Diserens, M. J., Harrison, M. F. A., \& Smith, A. C. H. 1984, J. Phys. B, 17, L621

Diserens, M. J., Smith, A. C. H., \& Harrison, M. F. A. 1988, J. Phys. B, 21, 2129

Djurić, N., Bell, E. W., \& Dunn, G. H. 1993a, Int. J. Mass Spectrom. Ion Processes, 123, 187

Djurić, N., Bell, E. W., Guo, X. Q., et al. 1993b, Phys. Rev. A, 47, 4786

Dolder, K. T. 1961, Proc. R. Soc. London A, 264, 367

Donets, E. D., \& Ovsyannikov, V. P. 1981, Sov. Phys.-JETP, 53, 466

Duponchelle, M., Khouilid, M., Oualim, E. M., Zhang, H., \& Defrance, P. 1997, J. Phys. B, 30, 729

Erickson, G. W. 1977, J. Phys. Chem. Ref. Data, 6, 831

Falk, R. A., \& Dunn, G. H. 1983, Phys. Rev. A, 27, 754

Falk, R. A., et al. 1980, unpublished, http://www-cfadc.phy. ornl.gov/xbeam/xbmintro.html

Falk, R. A., Dunn, G. H., Griffin, D. C., et al. 1981, Phys. Rev. Lett., 47, 494

Falk, R. A., Stefani, G., Camilloni, R., et al. 1983, Phys. Rev. A, 28, 91

Fontes, C. J., Sampson, D. H., \& Zhang, H. L. 1999, Phys. Rev. A, 59, 1329

Freund, R. S., Wetzel, R. C., Shul, R. J., \& Hayes, T. R. 1990, Phys. Rev. A, 41, 3575

Gao, H., Fang, D., Lu, F., et al. 1997, Nucl. Instr. Meth. Phys. Res. B, 132, 364.

Gorczyca, T. W., Pindzola, M. S., Griffin, D. C., \& Badnell, N. R. 1994, J. Phys. B, 27, 2399

Gregory, D. C., \& Crandall, D. H. 1982, unpublished, http://www-cfadc.phy.ornl.gov/xbeam/xbmintro.html

Gregory, D. C., \& Howald, A. M. 1986, Phys. Rev. A, 34, 97

Gregory, D. C., Dittner, P. F., \& Crandall, D. H. 1983, Phys. Rev. A, 27, 724

Gregory, D. C., Meyer, F. W., Müller, A., \& Defrance, P. 1986, Phys. Rev. A, 34, 3657

Gregory, D. C., Wang, L. J., Meyer, F. W., \& Rinn, K. 1987, Phys. Rev. A, 35, 3256

Gregory, D. C., Wang, L. J., Swenson, D. R., Sataka, M., \& Chantrenne, S. J. 1990, Phys. Rev. A, 41, 6512

Griffin, D. C., \& Pindzola, M. S. 1988, J. Phys. B, 21, 3253

Griffin, D. C., Bottcher, C., \& Pindzola, M. S. 1982a, Phys. Rev. A, 25, 154

Griffin, D. C., Bottcher, C., \& Pindzola, M. S. 1982b, Phys. Rev. A, 25, 1374

Griffin, D. C., Pindzola, M. S., \& Bottcher, C. 1984, J. Phys. B, 17, 3183

Griffin, D. C., Pindzola, M. S., \& Bottcher, C. 1987, Phys. Rev. A, 36, 3642

Gu, M. F. 2002, ApJ, 579, 103

Hartenfeller, U., Aichele, K., Harthiramani, D., et al. 1998, J. Phys. B, 31, 2999

Hartenfeller, U., Aichele, K., Harthiramani, D., et al. 1998, J. Phys. B, 31, 3013

Hayes, T. R., Wetzel, R. C., \& Freund, R. S. 1987, Phys. Rev. A, 35, 578

Hirayama, T., Oda, K., Morikawa, Y., et al. 1986, J. Phys. Soc. Japan, 55, 1411

Hooper, J. W., Lineberger, W. C., \& Bacon, F. M. 1966, Phys. Rev., 141, 165

Howald, A. M., \& Gregory, D. C. 1984 ,

http://www-cfadc.phy.ornl.gov/xbeam/xbmintro.html

Howald, A. M., Gregory, D. C., Meyer, F. W., et al. 1986, Phys. Rev. A, 33, 3779

Jacobi, J., Knopp, H., Schippers, S., et al. 2004, Phys. Rev. A, 70, 042717

Jalin, R., Hagemann, R., \& Botter, R. 1973, J. Chem. Phys., 59, 952

Kenntner, J., Linkemann, J., Badnell, N. R., et al. 1995, Nucl. Instr. Meth. Phys. Res. B, 98,142

Korchevoi, Yu. P., \& Przonski, A. M. 1967, Sov. Phys.-JETP, 24, 1089

LaGattuta, K. J., \& Hahn, Y. 1981, Phys. Rev. A, 24, 2273

Laghas, K., Reid, R. H. G., Joachain, C. J., \& Burke, P. G. 1999, J. Phys. B, 32, 1439

Landi, E., Del Zanna, G., Young, P. R., et al. 2006, ApJS, 162, 261

Lineberger, W. C. 1966, Phys. Rev., 141, 151

Linkemann, J., Müller, A., Kenntner, J., et al. 1995, Phys. Rev. Lett., 74, 4173

Loch, S. D., Colgan, J., Pindzola, M. S., et al. 2003, Phys. Rev. A, 67, 042714

Lotz, W. 1967a, ApJS, 14, 207

Lotz, W. 1967b, Z. Phys., 216, 241; 220, 466
Ma, C., Sporleder, C. R., \& Bonham, R. A. 1991, Rev. Sci. Inst., 62, 909

Man, K. F., Smith, A. C. H., \& Harrison, M. F. A. 1987a, J. Phys. B, 20, 2571

Man, K. F., Smith, A. C. H., \& Harrison, M. F. A. 1987b, J. Phys. B, 20, 5865

Man, K. F., Smith, A. C. H., \& Harrison, M. F. A. 1993, J. Phys. B, 26, 1365

Martin, S. O., Peart, B., \& Dolder, K. T. 1968, J. Phys. B, 1537

Matsumoto, A., Danjo, A., Ohtani, S., et al. 1990, J. Phys. Soc. Jpn, 59, 902

Mazzotta, P., Mazzitelli, G., Colafrancesco, S., \& Vittorio, N. 1998, A\&ASS, 133,403

McCallion, P., Shah, M. B., \& Gilbody, H. B. 1992a, J. Phys. B, 25, 1051

McCallion, P., Shah, M. B., \& Gilbody, H. B. 1992b, J. Phys. B, 25, 1061

McFarland, R. H., \& Kinney, J. D. 1965, Phys. Rev., 137, A1058

McGuire, E. J. 1977, Phys. Rev. A, 16, 62

Mitnik, D. M., Shaw, J. A., Pindzola, M. S., Griffin, D. C., \& Badnell, N. R. 1998, Computer Phys. Comm., 114, 368

Montague, R. G., \& Harrison, M. F. A. 1983, J. Phys. B, 16, 3045

Montague, R. G., Diserens, M. J., \& Harrison, M. F. A. 1984, J. Phys. B, 17, 2085

Montague, R. G., Harrison, M. F. A., \& Smith, A. C. H. 1984, J. Phys. B, 17, 3295

Moores, D. L., Golden, L. B., \& Sampson, D. H. 1980, J. Phys. B, 13, 385

Müller, A., Salzborn, E., Frodl, R., et al. 1980, J. Phys. B, 13, 1877

Müller, A., Huber, K., Tinschert, K., Becker, R., \& Salzborn, E. 1985a, J. Phys. B, 18, 2993

Müller, A., Tinschert, K., Aschenbach, C., Becker, R., \& Salzborn 1985b, J. Phys. B, 18, 3011

Müller, A., Hofmann, G., Tinschert, K., Weißbecker, B., \& Salzborn, E. 1990, Z. Phys. D, 15, 145

Mueller, D. W., et al. 1983, unpublished, http://www-cfadc.phy .ornl.gov/xbeam/xbmintro.html

Mueller, D. W., Morgan, T. J., Dunn, G. H., Gregory, D. C., \& Crandall, D. H. 1985, Phys. Rev. A, 31, 2905

Nagy, P., Skutlartz, A., \& Schmidt, V. 1980, J. Phys. B, 13, 1249

Nussbaumer, H., \& Storey, P. J. 1975, A\&A, 44, 321

O’Rourke, B., Currell, F. J., Kuramoto, H., et al. 2001, J. Phys. B, 34, 4003

Okuno, Y. 1971, J. Phys. Soc. Jpn, 31, 1189

Peart, B., \& Dolder, K. T. 1968a, J. Phys. B, 1, 240

Peart, B., \& Dolder, K. T. 1968b, J. Phys. B, 1, 872

Peart, B., \& Dolder, K. T. 1975, J. Phys. B, 8, 56

Peart, B., Martin, S. O., \& Dolder, K. T. 1969a, J. Phys. B, 2, 1176

Peart, B., Walton, D. S., \& Dolder, K. T. 1969b, J. Phys. B, 2, 1347

Peart, B., Thomason, J. W. G., \& Dolder, K. 1991a, J. Phys. B, 24, 489

Peart, B., Thomason, J. W. G., \& Dolder, K. 1991b, J. Phys. B, 24, 4453

Peart, B., Underwood, J. R. A., \& Dolder, K. 1989, J. Phys. B, 22, 2789

Pindzola, M. S., Griffin, D. C., Bottcher, C., et al. 1984, Phys. Rev. A, 29, 1749

Pindzola, M. S., Griffin, D. C., \& Bottcher, C. 1986a, Phys. Rev. A, 33, 3787

Pindzola, M. S., Griffin, D. C., \& Bottcher, C. 1986b, Phys. Rev. A, 34, 3668

Pindzola, M. S., Botcher, C., \& Griffin, D. C. 1987, J. Phys. B, 20, 3535

Pindzola, M. S., Griffin, D. C., Bottcher, C., Buie, M. J., \& Gregory, D. C. 1990, ORNL TM 11202, data available at http://dbshino.nifs.ac.jp/

Pindzola, M. S., Gorczyca, T. W., Badnell, N. R., et al. 1994, Phys. Rev. A, 49, 933

Qi, J. B., Chen, C. Y., Zhao, Y., Xia, H. N., \& Wang, Y. S. 2002, Phys. Rev. A, 65, 032720-1

Rachafi, S., Jureta, J., \& Defrance, P. 1987, Proc. 15th Int. Conf. on the Physics of Electronic and Atomic Collisions (Brighton), 378

Rachafi, S., Belić, D. S., Duponchelle, M., et al. 1991, J. Phys. B, 24, 1037

Ralchenko, Yu., Jou, F.-C., Kelleher, D. E., et al. 2006, NIST Atomic Spectra Database (version 3.1.0), [Online]. Available: http://physics.nist.gov/asd3 [2007, February 1]. National Institute of Standards and Technology, Gaithersburg, MD

Rakhovskii, V. I., \& Stepanov, A. M. 1969, High Temp., 7, 1001

Reed, K. J., \& Chen, M. H. 1996, Phys. Rev. A, 54, 2967

Reid, R. H. G., Bartschat, K., \& Burke, P. G. 1992, J. Phys. B, 25, 3175

Rejoub, R., \& Phaneuf, R. A. 2000, Phys. Rev. A, 61, 032706

Riahi, A., Laghdas, K., Reid, R. H. G., et al. 2001, J. Phys. B., 34, 175

Rinn, K., Gregory, D. C., Wang, L. J., Phaneuf, R. A., \& Mueller, A. 1987, Phys. Rev. A, 36, 595

Rogers, W. T., Stefani, G., Camilloni, R., et al. 1982, Phys. Rev. A, 25, 737

Sataka, M., Ohtani, S., Swenson, D., \& Gregory, D. C. 1989, Phys. Rev. A, 39, 2397

Shah, M. B., Elliott, D. S., \& Gilbody, H. B. 1987, J. Phys. B, 20, 3501

Shah, M. B., Elliott, D. S., McCallion, P., \& Gilbody, H. B. 1987, J. Phys. B, 21, 2751

Stenke, M., Hathiramani, D., Hofmann, G., et al. 1995a, Nucl. Instr. Meth. Phys. Res. B, 98, 138

Stenke, M., Aichele, K., Hathiramani, D., et al. 1995b, Nucl. Instr. Meth. Phys. Res. B, 98, 573

Stenke, M., Aichele, K., Hartenfeller, U., et al. 1999, J. Phys. B, 32, 3627 
Stephan, K., Helm, H., \& Mark, T. D. 1980, J. Chem. Phys., 73, 3763

Straub, H. C., Renault, P., Lindsay, B. G., Smith, K. A., \& Stebbings, R. F. 1995, Phys. Rev. A, 52, 1115

Shull, J. M., \& Van Steenberg, M. 1982, ApJS, 48, 95

Tawara, H. 2002, in Numerical Data and Functional Relationships in Science and Technology, ed. Y. Hahn, A. K. Pradhan, H. Tawara, H. L. Zhang, \& Y. Itakawa (gen. ed.), Landolt-Bornstein, 17B, 103

Tayal, S. S. 1994, Phys. Rev. A, 49, 2561

Teng, H., \& Xu, Z. 1996, Phys. Rev. A, 54, 444

Teng, H., Knopp, H., Ricz, S., et al. 2000, Phys. Rev. A, 61, 060704

Thomason, J. W. G., \& Peart, B. 1998, J. Phys. B, 31, L201

Thompson, J. S., \& Gregory, D. C. 1994, Phys. Rev. A, 50, 1377

Thompson, W. R., Shah, M. B., \& Gilbody, H. B. 1995, J. Phys. B, 28, 1321

Tinschert, K., Müller, A., Hofmann, G., et al. 1989a, J. Phys. B, 22, 531

Tinschert, K., Müller, A., Phaneuf, R. A., Hofmann, G., \& Salzborn, E. 1989b, J. Phys. B, 22, 1241

Vainshtein, L. A., Ochkur, V. I., Rakhovskii, V. I., \& Stepanov, A. M. 1972, Sov. Phys.-JETP, 34, 271

van Zoest, T., Knopp, H., Jacobi, J., et al. 2004, J. Phys. B, 37, 4387

Verner, D. A., \& Yakovlev, D. G. 1995, A\&AS, 109, 1225

Wang, L. J., Rinn, K., \& Gregory, D. C. 1988, J. Phys. B, 21, 2117
Wareing, J. B., \& Dolder, K. T. 1967, Proc. Phys. Soc., 91, 887

Wetzel, R. C., Baiocchi, F. A., Hayes, T. R., \& Freund, R. S. 1987, Phys. Rev. A, 35,559

Wong, K. L., Beiersdorfer, P., Chen, M. H., et al. 1993, Phys. Rev. A, 48, 2850

Woodruff, P. R., Hublet, M.-C., Harrison, M. F. A., \& Brook, E. 1978, J. Phys. B, 11,679

Yamada, I., Danjo, A., Hirayama, T., et al. 1988, J. Phys. Soc. Jpn, 57, 2699

Yamada, I., Danjo, A., Hirayama, T., et al. 1989a, J. Phys. Soc. Jpn, 58, 1585

Yamada, I., Danjo, A., Hirayama, T., et al. 1989b, J. Phys. Soc. Jpn, 58, 3151

Younger, S. M. 1981a, Phys. Rev., 23, 1138

Younger, S. M. 1981b, Phys. Rev., 24, 1272

Younger, S. M. 1981c, Phys. Rev., 24, 1278

Younger, S. M. 1982a, JSQRT, 27, 541

Younger, S. M. 1982b, Phys. Rev. A, 26, 3177

Zapesochnyi, I. P., \& Aleksakhin, I. S. 1969, Sov. Phys. JETP, 28, 41

Zeijlmans van Emmichoven, P. A., Bannister, M. E., Gregory, D. C., et al. 1993, Phys. Rev. A, 47, 2888

Zhang, H., Cherkani-Hassani, S., Bélenger, C., Duponchelle, M., et al. 2002, J. Phys. B, 35, 3829

Zhang, Y., Reddy, C. B., Smith, R. S., et al. 1991, Phys. Rev. A, 44, 4368

Zhang, Y., Reddy, C. B., Smith, R. S., et al. 1992, Phys. Rev. A, 45, 2929 